\title{
Engineering Bimetallic Centers in Porous Silicate Materials for Hydrogenation of Furfural at Lower Temperature
}

$$
\text { Jiahui Ye, }{ }^{a, b} \text { Siqi Huang }{ }^{a, b} \text { and Chuan-De } \mathrm{Wu}^{* a}
$$

${ }^{a}$ State Key Laboratory of Silicon Materials, Department of Chemistry, Zhejiang University, Hangzhou 310027, P. R. China.

${ }^{b}$ School of Chemical Engineering and Light Industry, Guangdong University of Technology, Guangzhou 510006, P. R. China 


\section{Experimental}

\section{Materials and methods}

All reagents were commercially obtained without further purification. $\mathrm{Cu}\left(\mathrm{NO}_{3}\right)_{2} \cdot 3 \mathrm{H}_{2} \mathrm{O}(>99 \%)$, $\mathrm{Cu}$ metal (>99.9\%), dodecane and tetraethyl orthosilicate (TEOS, $>98 \%$ ) were supplied by Macklin Chemical Reagent Company. Furfural (>99.8\%), $\mathrm{Co}\left(\mathrm{NO}_{3}\right)_{2} \cdot 6 \mathrm{H}_{2} \mathrm{O}(>99 \%)$, benzoic acid (>99.5\%), N,N-dimethylformamide (DMF, >99.5\%), methanol (>99.5\%), ethanol (>99.5\%), propanol $(>99.5 \%)$, isopropanol $(>99.5 \%)$, n-butanol $(>99.4 \%)$ and isobutanol $(>99.0 \%)$ were available from Aladdin-Reagent. Deionized (DI) water was used in all experimental processes. Nitrogen gas sorption isotherms were measured at $77 \mathrm{~K}$ on Micromertics ASAP-2460. The specific surface areas were calculated by employing the Brunauer-Emmett-Teller (BET) equation in the pressure range of $p / p_{0}=0.05-0.30$. The pore size distributions were calculated by using nonlocal density functional theory (NLDFT) method. Powder X-ray diffraction (PXRD) patterns were collected using Tongda TZL-30F diffractometer with Nickel-filtered $\mathrm{Cu} K \alpha$ radiation $(\lambda=1.5418$ $\AA$ ) in a $2 \theta$ range of $5-80^{\circ}$ at $40 \mathrm{kV}$ and $40 \mathrm{~mA}$, and the step size and scan speed were $0.04^{\circ}$ and 1.2 \%/min, respectively. The morphology of solid materials was studied using a JSM-7500F (JEOL, Japan) scanning electron microscope (SEM) and a Tecnai-G20 (FEI) transmission electron microscope (TEM). Fourier transform infrared (FT-IR) spectroscopy was performed on TENSOR II (Bruker). X-ray photoelectron spectroscopy (XPS) measurements were carried out on Escalab $250 \mathrm{Xi}$, and the calibration was performed using $\mathrm{C} 1 \mathrm{~s}$ binding energy at $284.8 \mathrm{eV}$. Inductively coupled plasma optical emission spectroscopy (ICP-OES) was carried out on iCAP-6500 (Thermo Scientific). The solid samples (ca. $50 \mathrm{mg}$ ) were dissolved in $\mathrm{HF}$ aqueous solution (40\%) to prepare the aqueous solutions of the samples for ICP-OES measurements. 


\section{Synthesis of PMS-2}

By employing sol-gel method, small coordination molecules could be highly dispersed or appropriately aggregated in the PMS precursors. Typically, benzoate acid (2.00 g, $16.38 \mathrm{mmol})$ was dissolved in $10 \mathrm{~mL} \mathrm{DMF}$, and $\mathrm{Cu}\left(\mathrm{NO}_{3}\right)_{2} \cdot 3 \mathrm{H}_{2} \mathrm{O}(0.49 \mathrm{~g}, 2.05 \mathrm{mmol})$ and $\mathrm{Co}\left(\mathrm{NO}_{3}\right)_{2} \cdot 6 \mathrm{H}_{2} \mathrm{O}(0.60$ g, $2.05 \mathrm{mmol}$ ) were dissolved in $5 \mathrm{~mL} \mathrm{H}_{2} \mathrm{O}$, which were subsequently mixed under stirring at room temperature. After reacting at room temperature for $30 \mathrm{~min}$, TEOS (3.41 g, $16.38 \mathrm{mmol})$ was dropwise added into the mixed solution under vigorous stirring. The resulting clean solution was transformed into Teflon-lined autoclave to further react at $100{ }^{\circ} \mathrm{C}$ for $20 \mathrm{~h}$. The resulting sol-gel was rinsed with deionized $\mathrm{H}_{2} \mathrm{O}$, crudely mashed and then dried at $65{ }^{\circ} \mathrm{C}$ in vacuum oven. The PMS-2 precursor was obtained by grinding the as-obtained purple solid, which was subsequently annealed in Ar atmosphere at $700{ }^{\circ} \mathrm{C}$ for $6 \mathrm{~h}$, denoted the resulting material as PMS-2. To make comparisons, we also synthesized a series of control catalysts with different $\mathrm{Cu} / \mathrm{Co}$ ratios under the identical conditions, denoted as PMS-2- $\mathrm{Cu}(\mathrm{Cu}: \mathrm{Co}=1: 0), \mathrm{PMS}-2-\mathrm{Cu}_{3} \mathrm{Co}(\mathrm{Cu}: \mathrm{Co}=3: 1)$, PMS$2-\mathrm{CuCo}_{3}(\mathrm{Cu}: \mathrm{Co}=1: 3)$ and PMS-2-Co $(\mathrm{Cu}: \mathrm{Co}=0: 1)$.

\section{Catalytic Property Evaluation}

Hydrogenation of furfural was performed in a $50 \mathrm{~mL}$ autoclave lined with polytetrafluoroethylene (PTFE). Furfural (2.5 mmol), solvent $(10 \mathrm{~mL})$, dodecane (internal standard; $50 \mu \mathrm{L})$ and PMS-2 ( $0.1 \mathrm{mmol}$ based on the metal contents of $\mathrm{Cu}$ and $\mathrm{Co}$ ) were added into the autoclave, and purged with 1.0 MPa $\mathrm{H}_{2}$ five times for $20 \mathrm{~min}$ to remove the dissolved air. The autoclave was heated to the required temperature under stirring $(600 \mathrm{rpm})$. After reaction, the reactor was immersed in an ice-water bath immediately. The solid catalyst was recovered by centrifugation, and the products 
were identified by GC-MS (ATOMX-7890B-5977B, Agilent) and analyzed by GC (9790II, Fuli). Furfural conversion and product selectivity were calculated by using the following equations.

$$
\begin{aligned}
& \text { Conversion }=\frac{n_{\text {furfural, } \text { initial }}-n_{\text {furfural }}}{n_{\text {furfural, } \text {, initial }}} \times 100 \%
\end{aligned}
$$

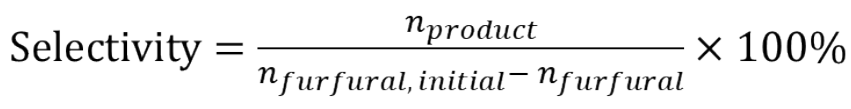

Turnover frequency (TOF) is the amount of converted furfural over per mol of metal species (the amount of $\mathrm{Cu}+\mathrm{Co}$ ) per hour. In the recycling catalytic experiments, the solid catalyst was collected by centrifugation, washed with methanol, and dried at $40{ }^{\circ} \mathrm{C}$ overnight in a vacuum oven to remove the adsorbates.

\section{Theoretical Calculations}

The first-principles calculations were performed with DFT using projector augmented wave within PBE, implemented by Vienna ab-initio simulation package (VASP). ${ }^{\mathrm{S} 1 \mathrm{S2} 2}$ The lattice constant and cohesive energy of the $\mathrm{Cu}$ unit cell are $3.61 \AA$ and $3.47 \mathrm{eV} /$ atom, which are consistent with the literature (3.63 $\AA$ and $3.48 \mathrm{eV} /$ atom), respectively. ${ }^{\mathrm{S} 3} \mathrm{The} \mathrm{Cu}(110)$ surface was structured by six layers, containing 36 atoms. A $450 \mathrm{eV}$ plane-wave cutoff and Monkhorst-Pack k-point grid of (5 $\times 5 \times 1)$ were used for $\mathrm{Cu}(110)$ and $\mathrm{Cu}-\mathrm{Co}$ alloy. ${ }^{\mathrm{S} 4}$ Spin-polarization was considered in all cases. The bottom two layers were fixed, and the other atoms were fully relaxed until the energy and residual force were less than $1 \times 10^{-4} \mathrm{eV}$ and $0.01 \mathrm{eV} / \AA$, respectively. The energy barriers for furfural hydrogenation were estimated using the climbing image nudged elastic band (CI-NEB) method in VASP. ${ }^{\mathrm{S} 5}$ The energies and total force convergence criteria for all atoms of images were below $1 \times 10^{-4} \mathrm{eV}$ and $0.1 \mathrm{eV} / \AA$, respectively. A Monkhorst-Pack grid $(15 \times 15 \times 1)$ was used for 
the models used in the Bader Charge analysis, projected density of states (PDOSs) and charge density analysis. ${ }^{\mathrm{S} 6, \mathrm{~S} 7}$ 


\section{Scheme and Figures}

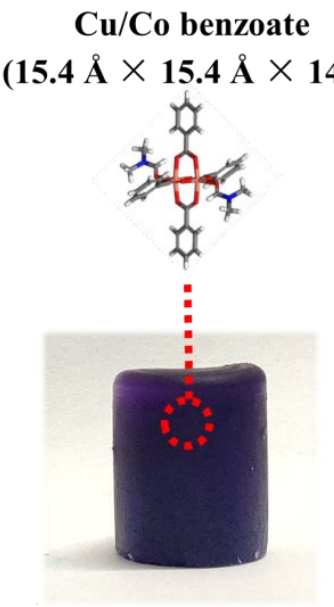

PMS-2 Precursor

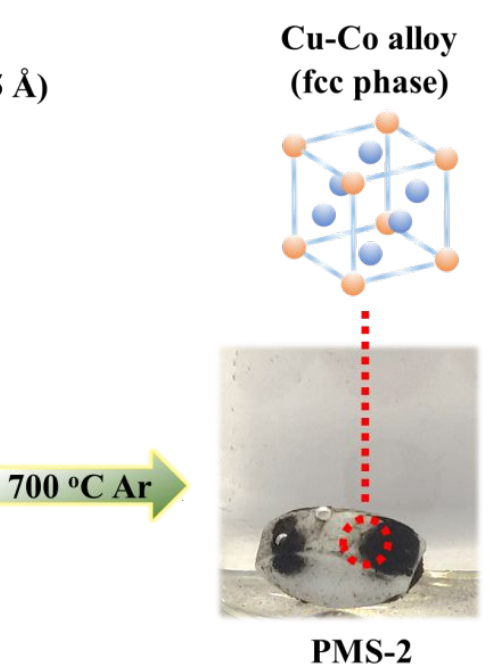

PMS-2

Scheme S1. Schematic illustration of paddle-wheel $\mathrm{Cu} / \mathrm{Co}$-benzoate molecule and $\mathrm{Cu}-\mathrm{Co}$ bimetallic species encapsulated in the PMS-2 precursor and PMS-2, respectively.

The $\mathrm{Cu} / \mathrm{Co}$-benzoate paddle-wheel molecule, with dimensions of $15.4 \AA \times 15.4 \AA \times 14.5 \AA$, is built from the coordination between two metal ions and four carboxylate ligands, as shown in Scheme $\mathrm{S} 1$. Unlike the infinite network of $\mathrm{Cu} / \mathrm{Co}$-based MOFs, $\mathrm{Cu} / \mathrm{Co}$ metal ions only coordinate to terminal benzoates in $\mathrm{Cu} / \mathrm{Co}$-benzoate, which are easily dispersed in silica gel. After treating in Ar atmosphere at $700{ }^{\circ} \mathrm{C}$, the resulting PMS-2 material is magnetic. 


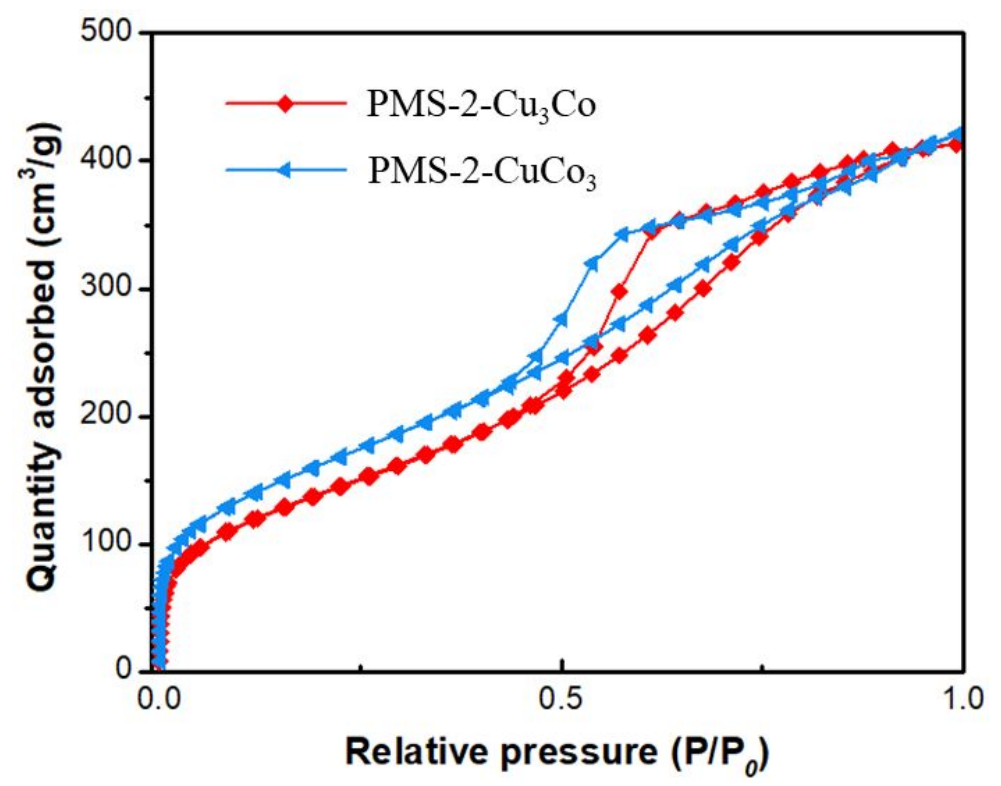

Figure S1. $\mathrm{N}_{2}$ adsorption/desorption isotherms of $\mathrm{PMS}-2-\mathrm{Cu}_{3} \mathrm{Co}$ and $\mathrm{PMS}-2-\mathrm{CuCo}_{3}$. 

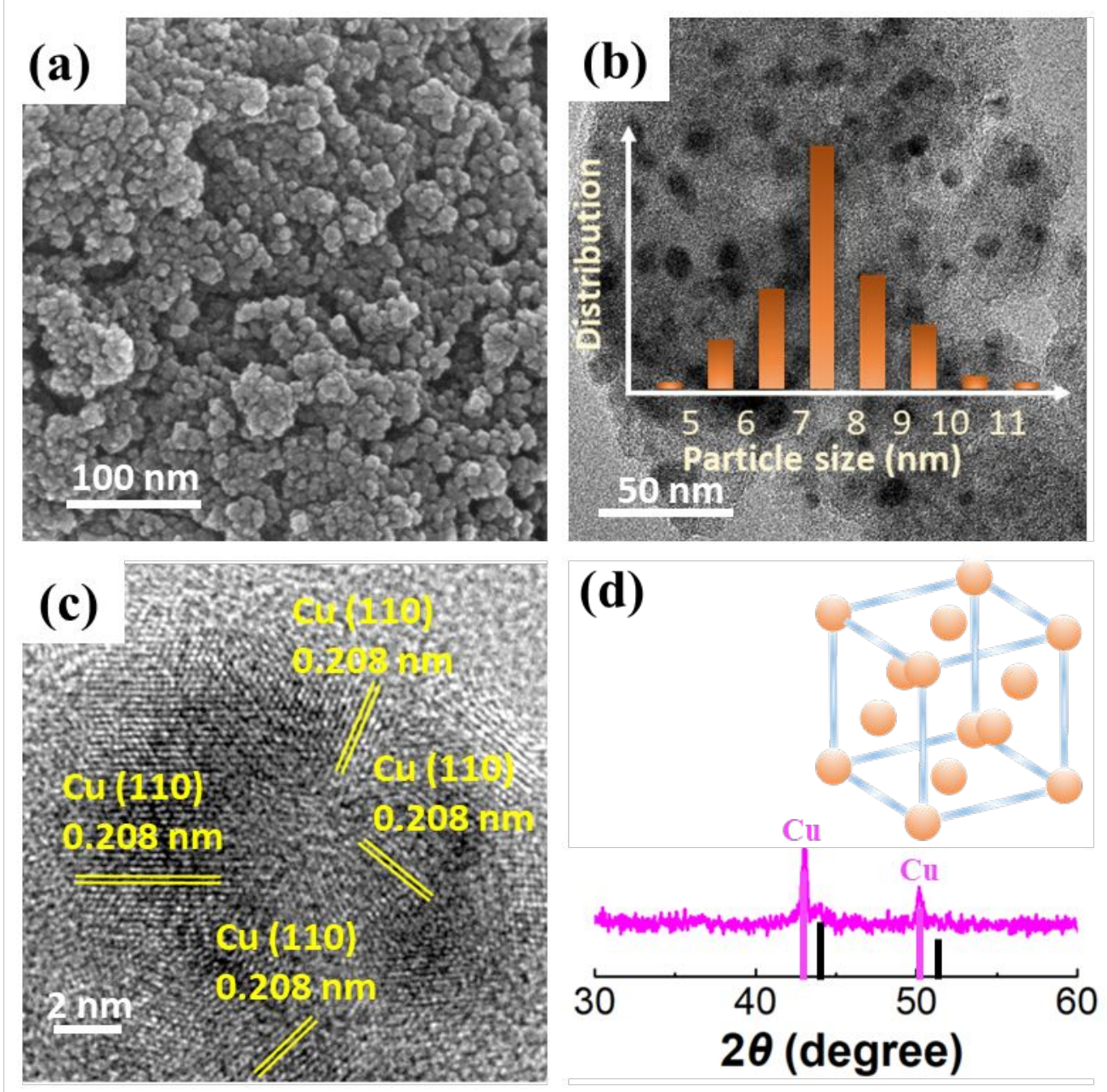

Figure S2. (a) SEM, (b) HRTEM (the insert shows metal particle size distribution), (c) lattice fringe, and (d) PXRD pattern for PMS-2-Cu. 

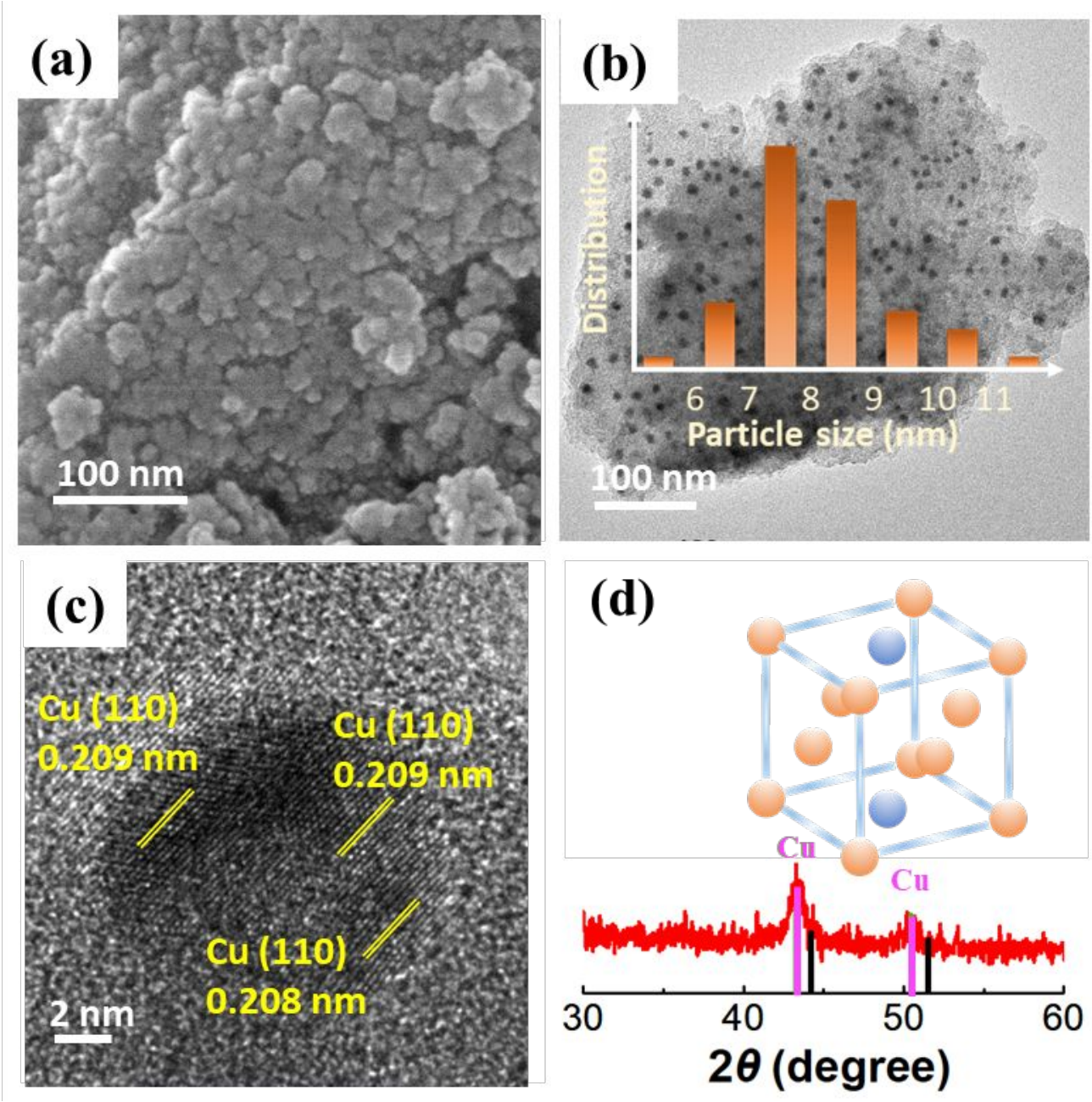

Figure S3. (a) SEM, (b) HRTEM (the insert shows metal particle size distribution), (c) lattice fringe, and (d) PXRD pattern for PMS-2-Cu 3 Co. 

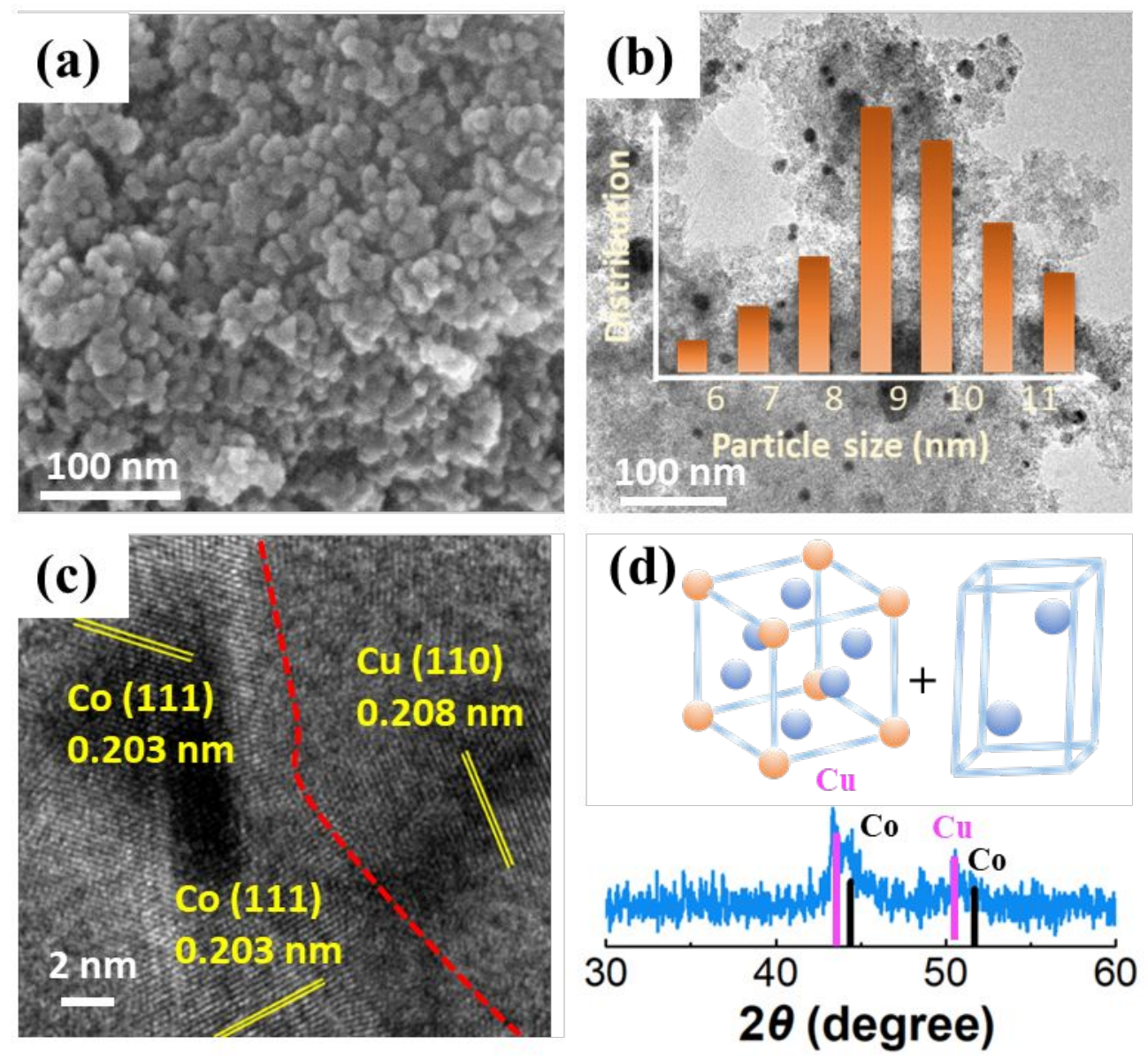

Figure S4. (a) SEM, (b) HRTEM (the insert shows metal particle size distribution), (c) lattice fringe, and (d) PXRD pattern for PMS-2-CuCo 3 . 

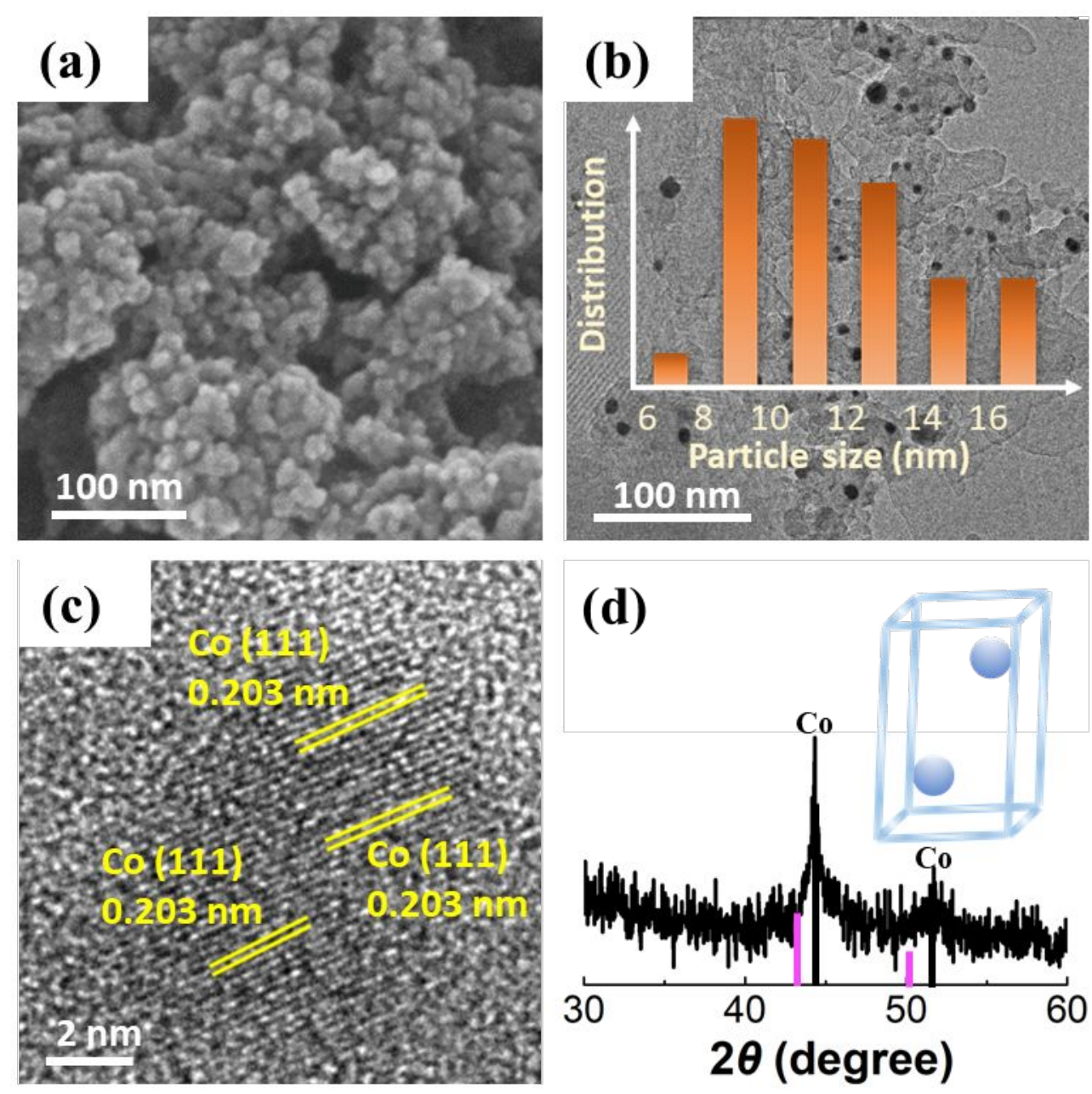

Figure S5. (a) SEM, (b) HRTEM (the insert shows metal particle size distribution), (c) lattice fringe, and (d) PXRD pattern for PMS-2-Co. 


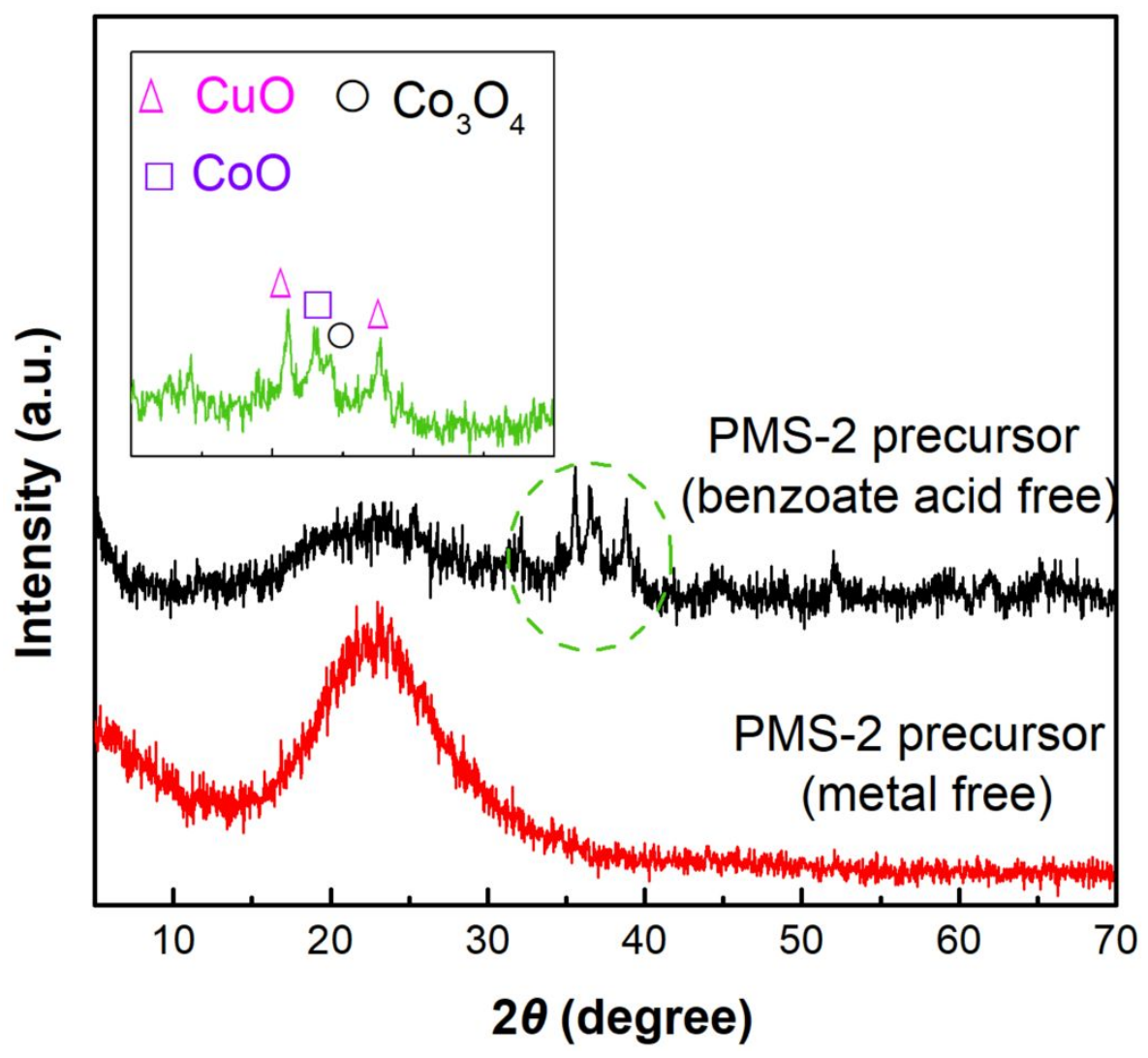

Figure S6. PXRD profiles of the PMS-2 precursors in the absence of metal salt or benzoate acid. In the absence of benzoic acid, the $\mathrm{Cu} / \mathrm{Co}$ components were easily oxidized into $\mathrm{CuO} / \mathrm{CoO} / \mathrm{Co}_{3} \mathrm{O}_{4}$ during hydrothermal process. 

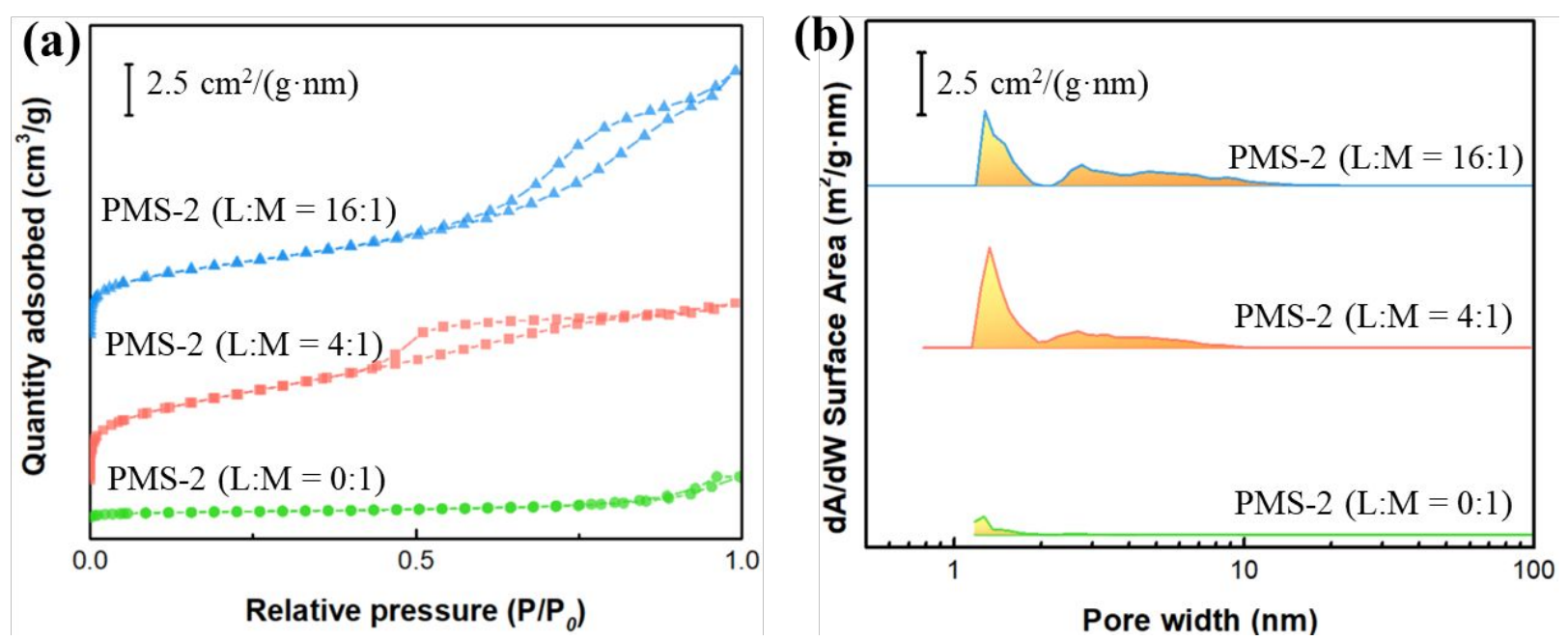

Figure S7. (a) $\mathrm{N}_{2}$ adsorption-desorption isotherms and (b) DFT pore size distributions for PMS-2 with different ligand/metal (L:M) ratios. 


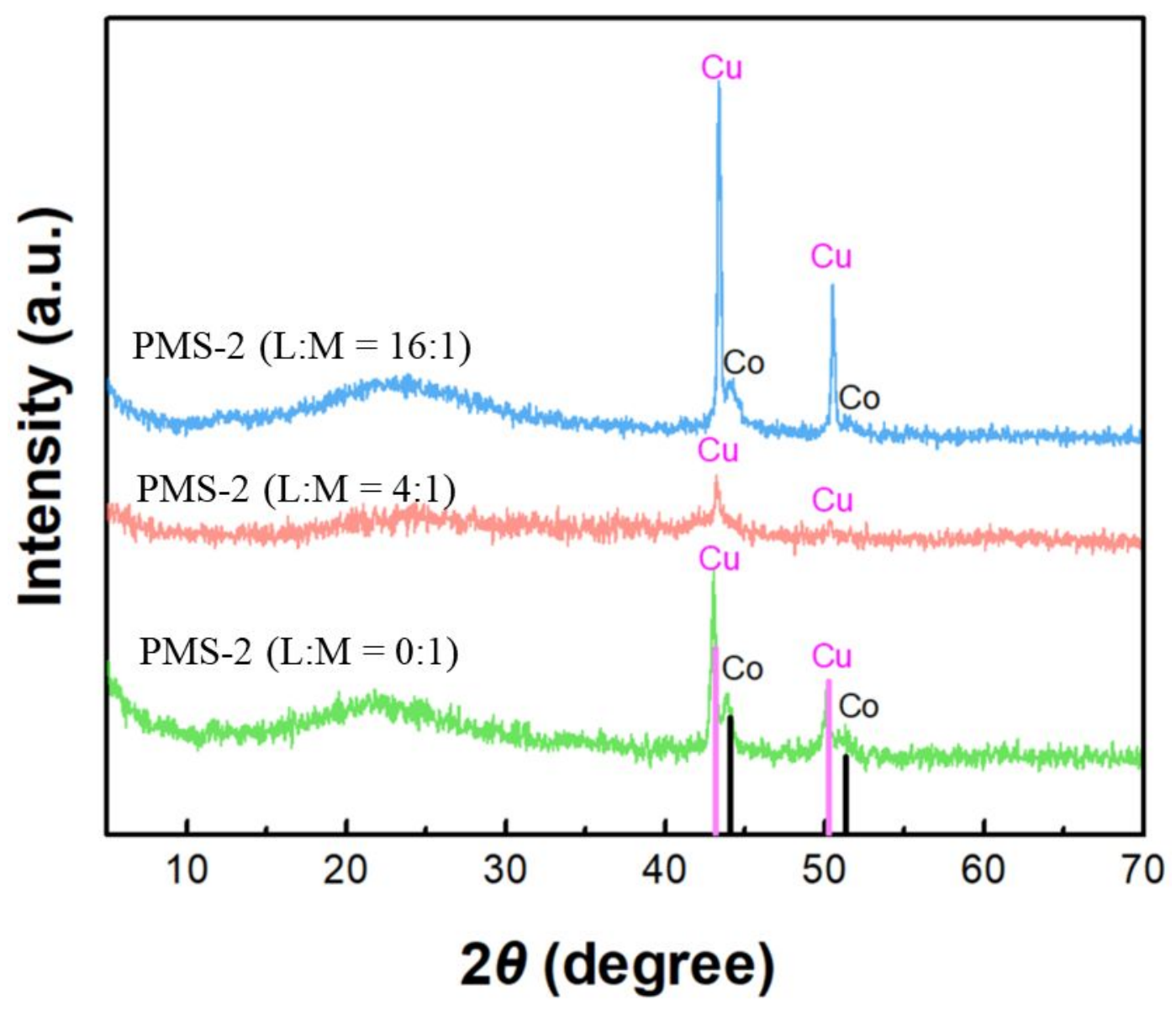

Figure S8. PXRD profiles of PMS-2 with different benzoate acid/metal (L:M) ratios.

The benzoic acid/metal ratio is one of the vital factors to determine the pore distribution and active components. In the absence of benzoate acid, $\mathrm{Cu}^{2+} / \mathrm{Co}^{2+}$ ions are easily oxidized into high oxidation states during hydrothermal processes, and the surface area of resulting PMS material is of only $57 \mathrm{~m}^{2} \mathrm{~g}^{-1}$ (Table S3). When the benzoate/metal ratio was increased to 16:1, the surface area of resulting PMS material decreased from 675 to $498 \mathrm{~m}^{2} \mathrm{~g}^{-1}$, while the hysteresis loop shifted to a higher range of $\mathrm{P} / \mathrm{P}_{0}=0.55-0.95$, compared with those of PMS-2. PXRD profiles show that the diffraction intensity for Co metallic species is also variable, indicating that benzoate/metal ratio is a parameter to control the porosity and the constituent of bimetallic active site for the PMS materials (Figure S8). 

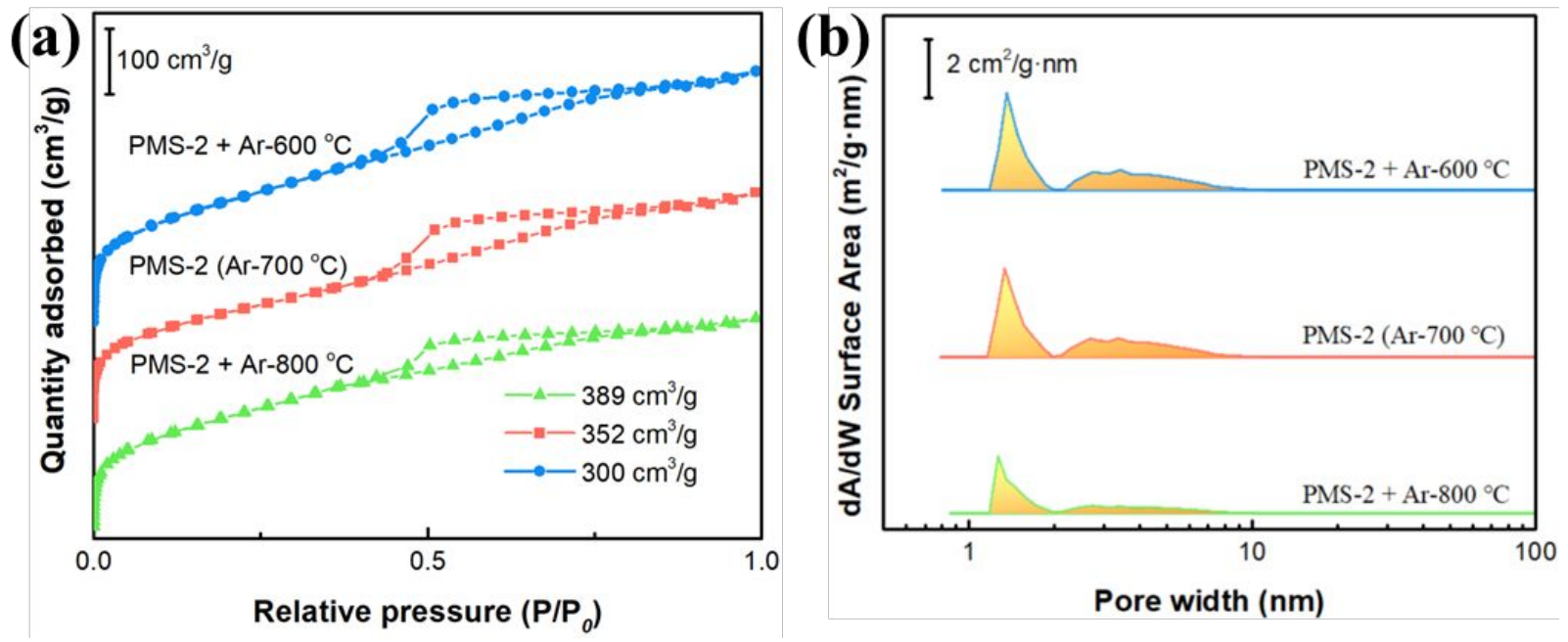

Figure S9. (a) $\mathrm{N}_{2}$ adsorption-desorption isotherms and (b) DFT pore size distributions for PMS-2 annealed at different temperatures. 


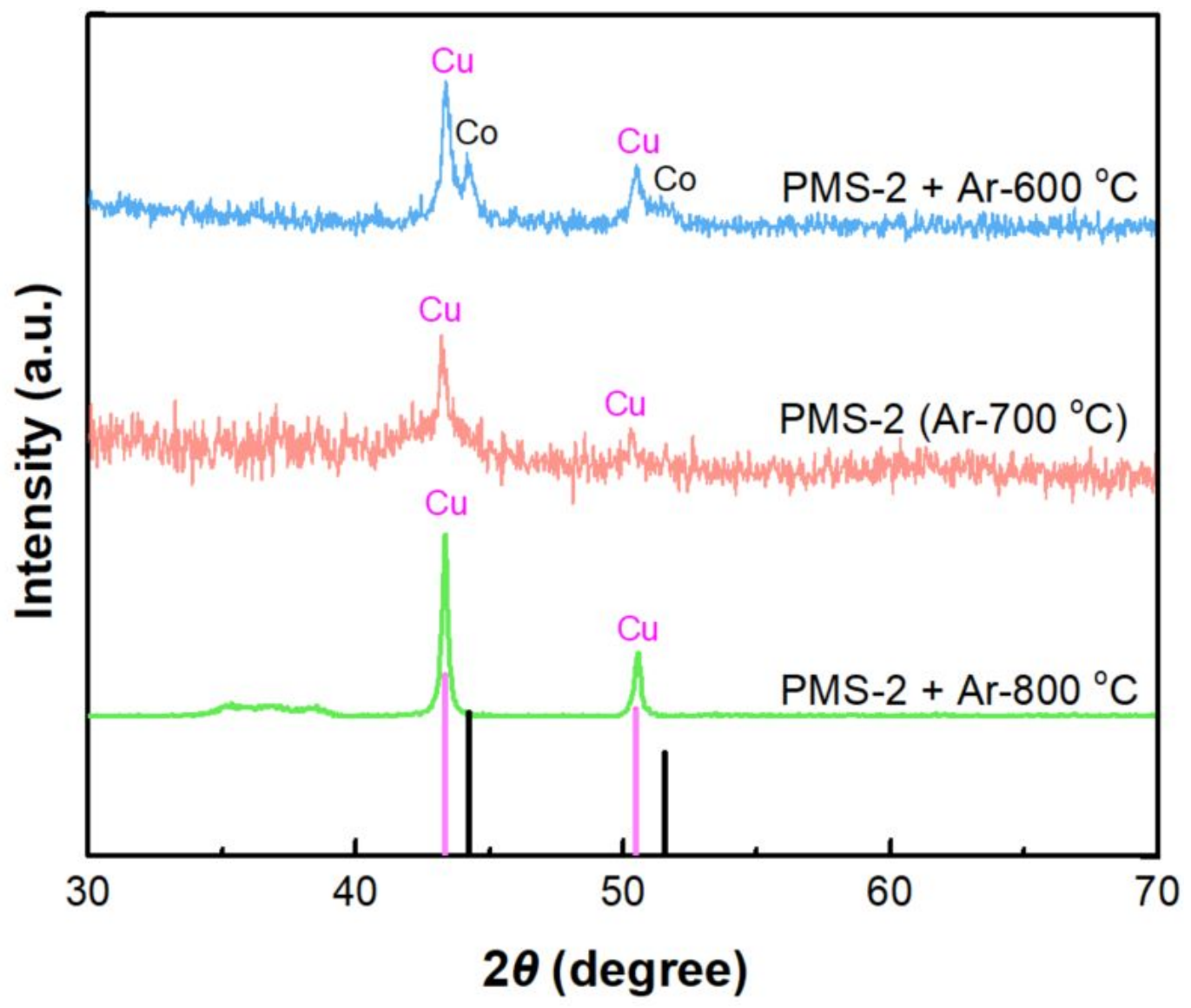

Figure S10. PXRD profiles for PMS-2 annealed at different temperatures. When the annealing temperature was increased from 600 to $800{ }^{\circ} \mathrm{C}$, discrete $\mathrm{Co}$ and $\mathrm{Cu}$ metallic species was gradually alloyed. 

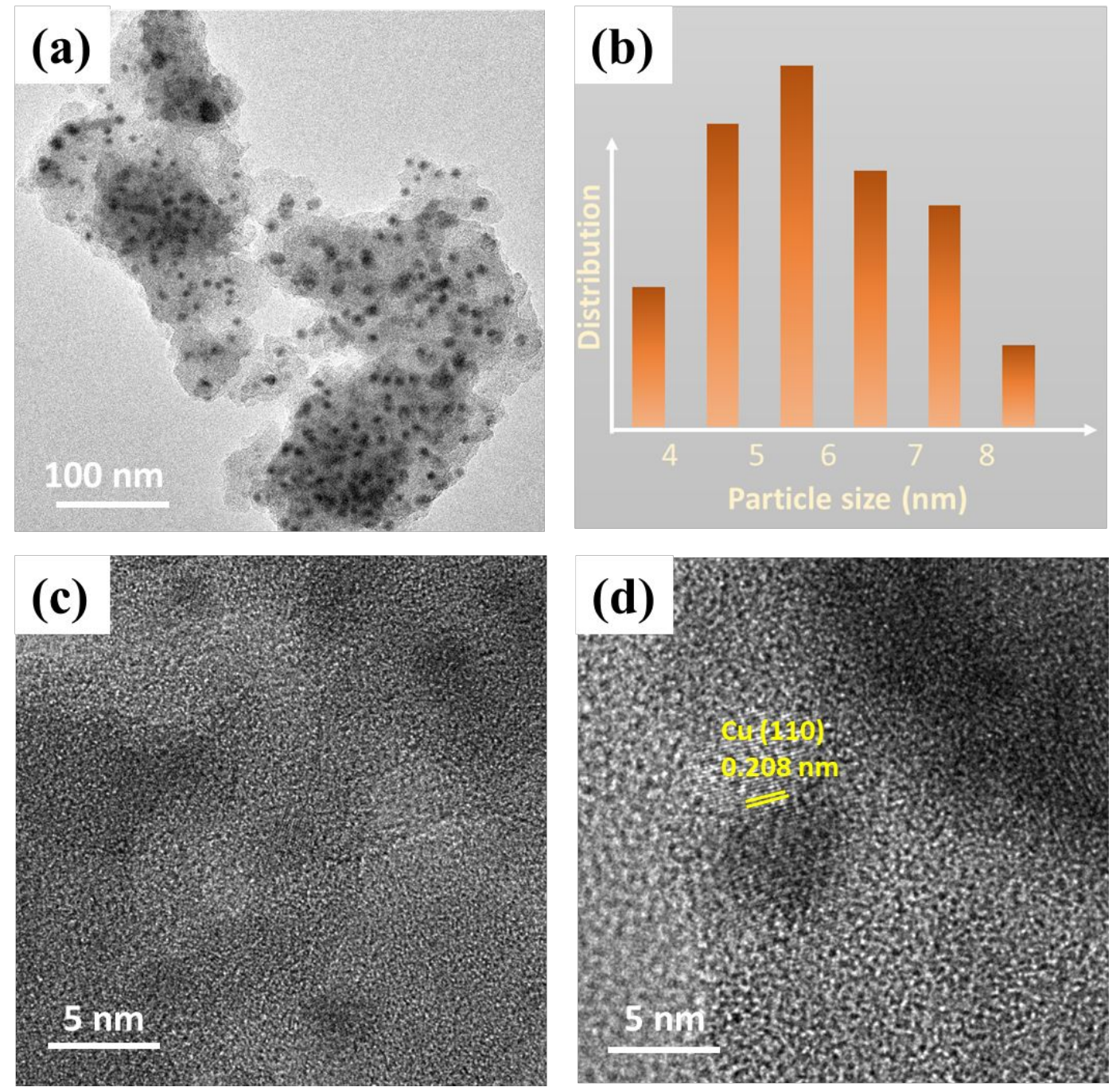

Figure S11. HRTEM images and metal particle size distribution for PMS-2 annealed in Ar atmosphere at $600{ }^{\circ} \mathrm{C}$. 

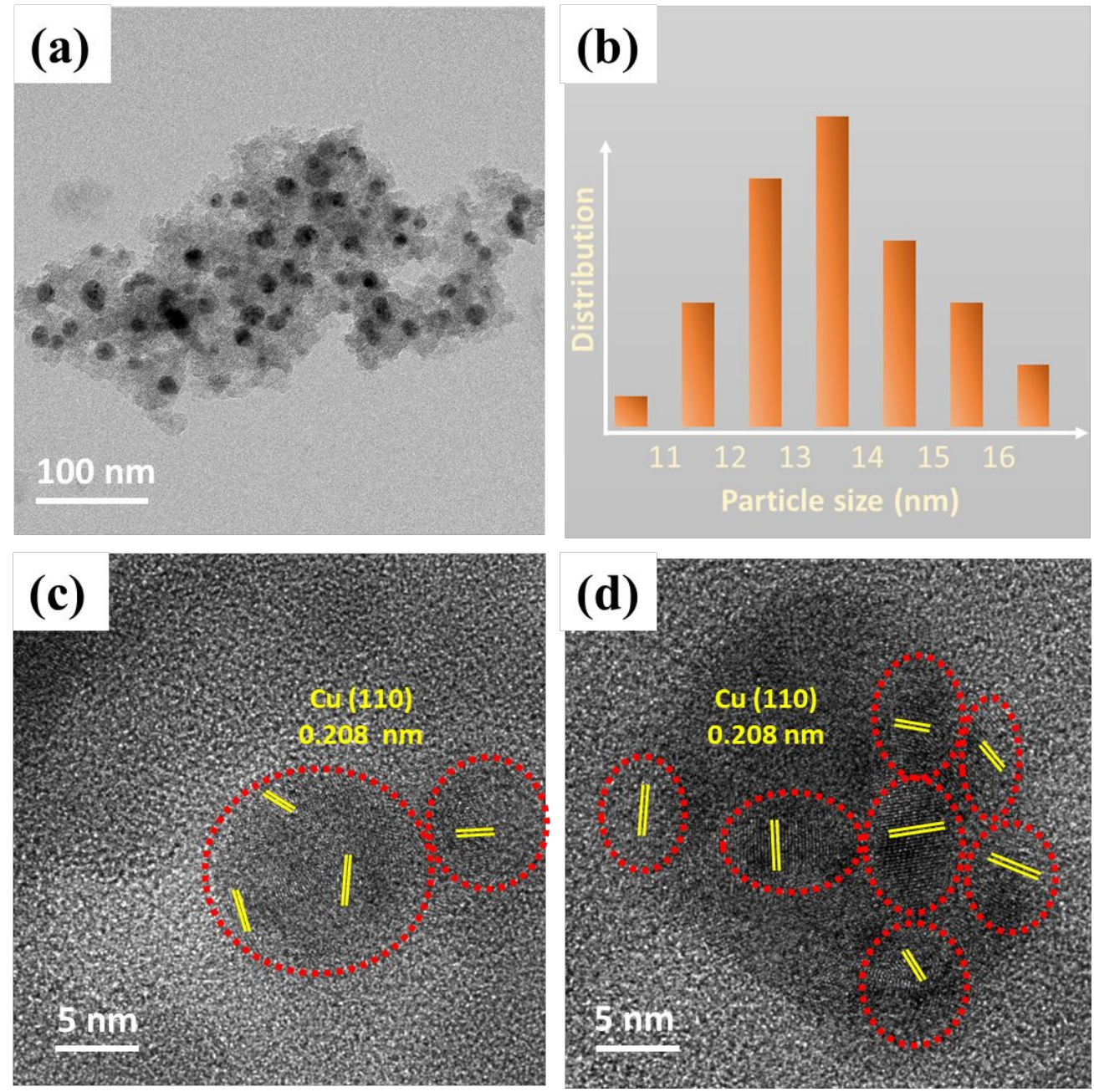

Figure S12. HRTEM images and metal particle size distribution for PMS-2 annealed in Ar atmosphere at $800{ }^{\circ} \mathrm{C}$. Combined with Figure S11, the HRTEM images indicate that high annealing temperature would lead to formation of large metal particles. 


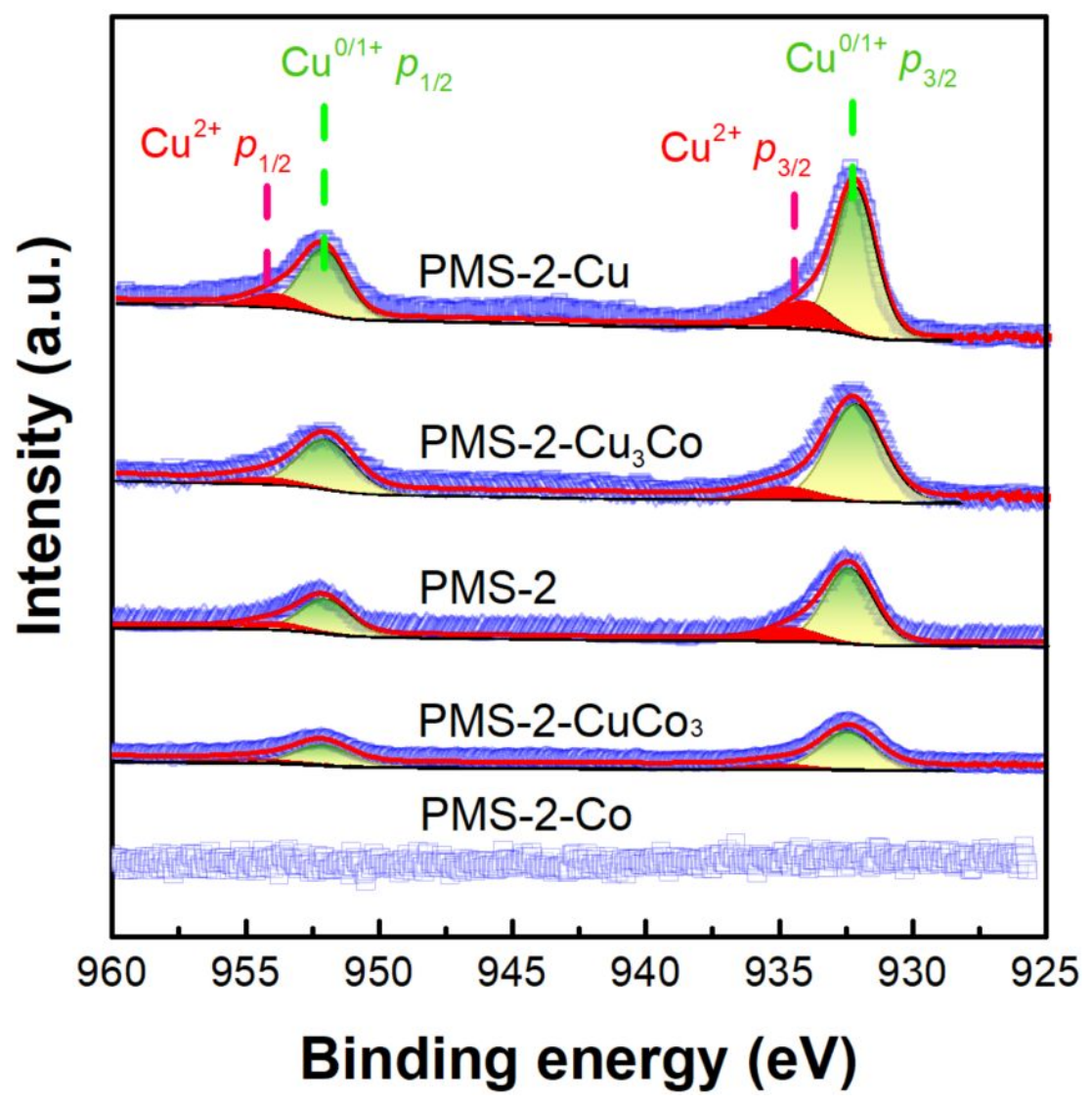

Figure S13. High resolution $\mathrm{Cu} 2 p$ XPS spectra for different PMS materials. 


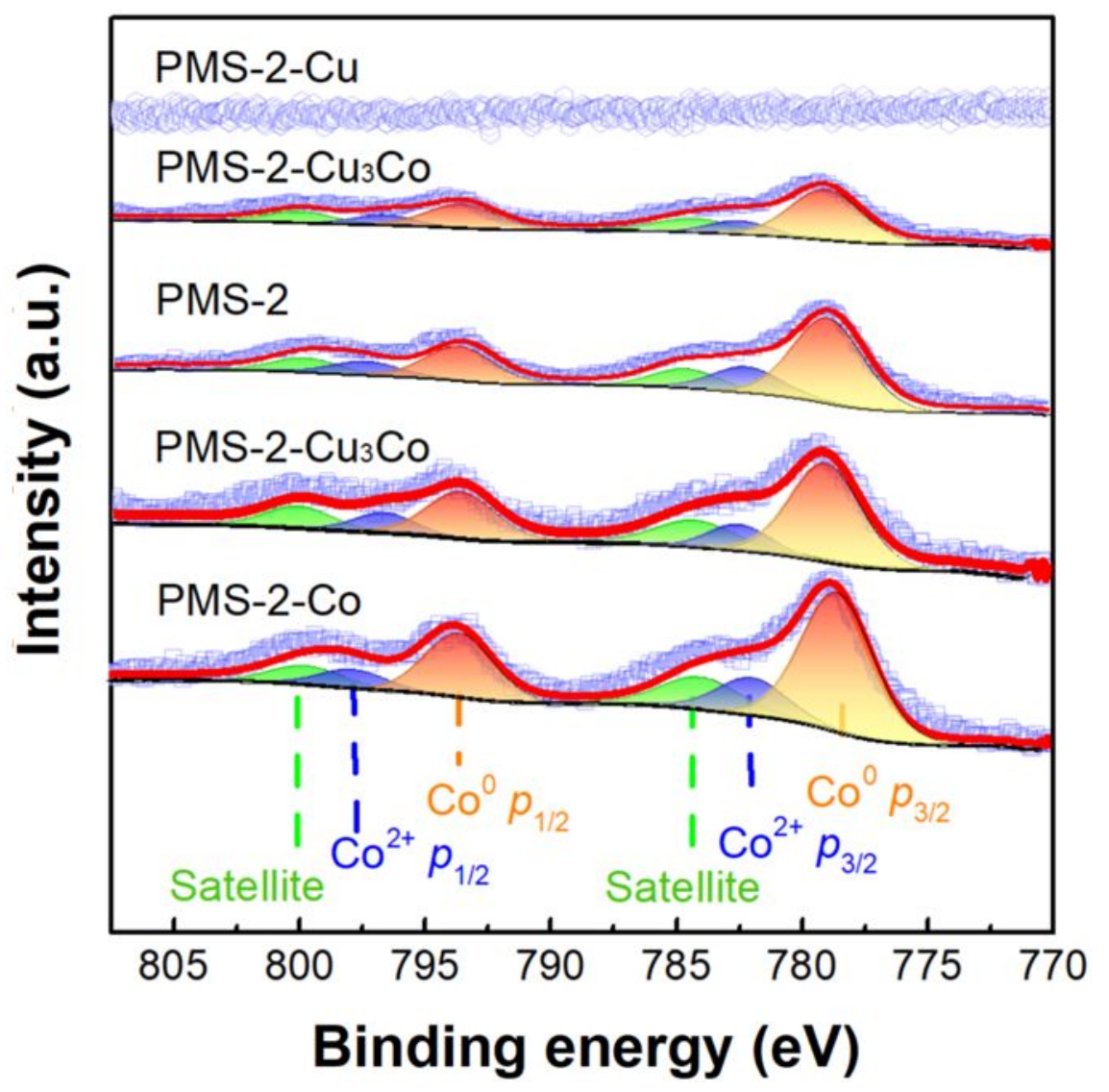

Figure S14. High resolution Co $2 p$ XPS spectra for different PMS-2 materials.

As shown in Figure $\mathrm{S} 13$, the binding energies of $\mathrm{Cu}^{0} / \mathrm{Cu}^{+} 2 p_{3 / 2}$ and $2 p_{1 / 2}$ locate at $\sim 932.4$ and $\sim 952.1 \mathrm{eV}$, respectively. ${ }^{\mathrm{S} 8, \mathrm{~S} 9}$ The binding energies of $\mathrm{Co}^{0} 2 p_{3 / 2}$ and $2 p_{1 / 2}$ appear at $\sim 778.5$ and $\sim 793.4 \mathrm{eV}$, while those of $\mathrm{Co}^{2+} 2 \mathrm{p}_{3 / 2}$ and $2 \mathrm{p}_{1 / 2}$ locate at $\sim 781.8$ and $\sim 797.7 \mathrm{eV}$, respectively (Figure S14). ${ }^{\mathrm{S} 10, \mathrm{~S} 11}$ When tuning the $\mathrm{Cu} / \mathrm{Co}$ ratio in the precursors, the $\mathrm{Cu}^{0 / 1+} / \mathrm{Cu}^{2+}$ and $\mathrm{Co}^{0} / \mathrm{Co}^{2+}$ ratios are not obviously changed (Tables S5 and S6). 

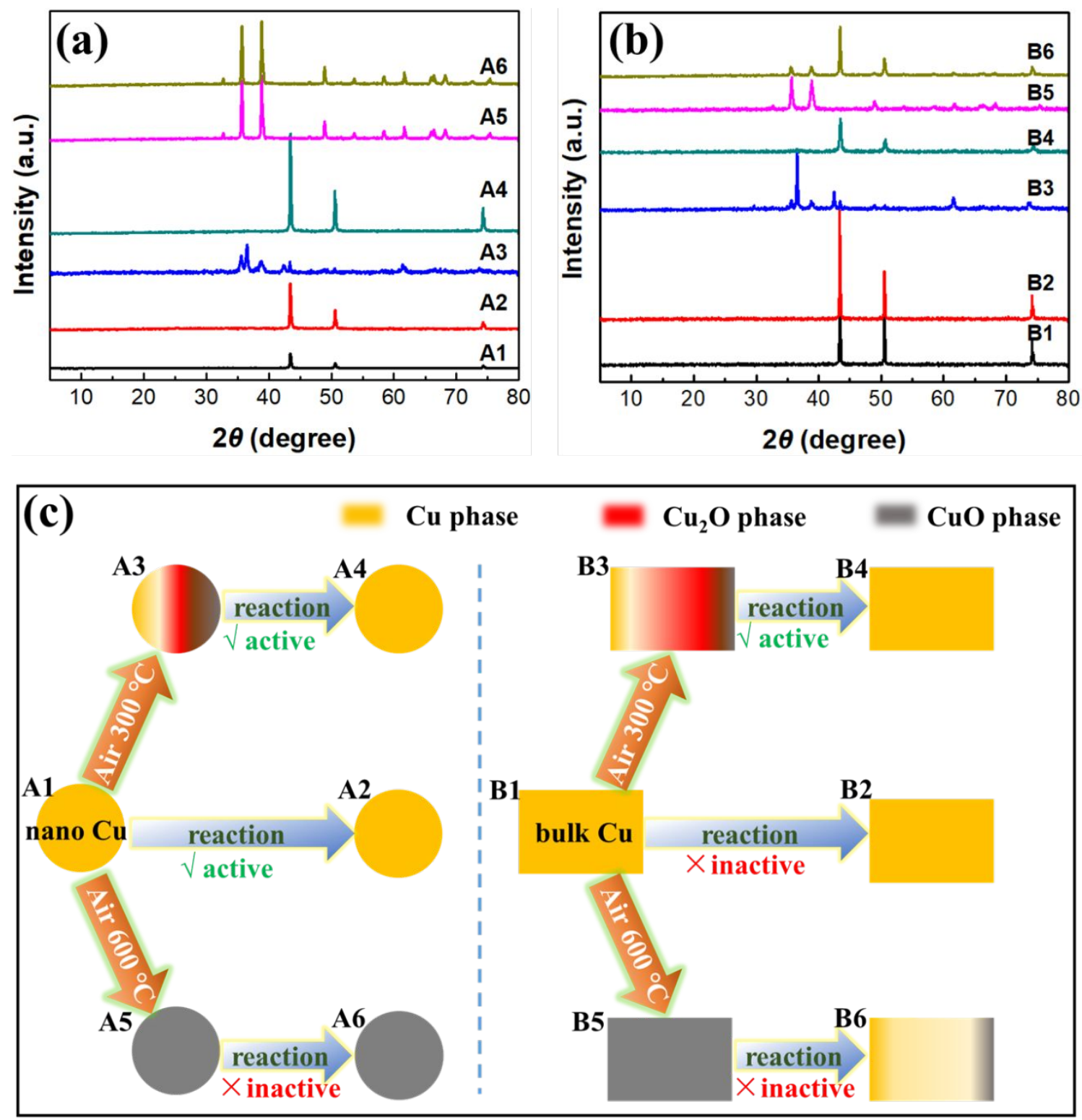

Figure S15. PXRD patterns of (a) nano $\mathrm{Cu}$ and (b) bulk $\mathrm{Cu}$ treated under various conditions and after catalysis, and (c) phase changes and catalytic behaviors. Reaction conditions: $1.0 \mathrm{mmol}$ furfural, $1.5 \mathrm{mmol} \mathrm{Cu}$ catalyst, $10 \mathrm{~mL}$ methanol, $1 \mathrm{MPa} \mathrm{H}_{2}, 140{ }^{\circ} \mathrm{C}, 6 \mathrm{~h}$. The catalytic results are shown in Table S9.

We invested the active sites by comparing the catalytic behaviors of nano $\mathrm{Cu}(60-100 \mathrm{~nm})$ and bulk $\mathrm{Cu}$ powder (micron scale). Figure S15a and 15b shows the PXRD profiles of different samples, while the data are shown in Table S9. The particle size of $\mathrm{Cu}$ metal species is an important factor to determine the catalytic properties; nano $\mathrm{Cu}$ delivered furfural conversion of $66 \%$, while 
bulk $\mathrm{Cu}$ powder is inactive for furfural hydrogenation under the identical catalytic conditions. After treated in air at $300{ }^{\circ} \mathrm{C}$, the crystal phase of $\mathrm{Cu}$ was transferred into $\mathrm{Cu} / \mathrm{Cu}_{2} \mathrm{O} / \mathrm{CuO}$ for both kinds of $\mathrm{Cu}$ metal samples. The partially oxidized nano $\mathrm{Cu}$ metal resulted in slightly decreased furfural conversion from 66 to $51 \%$. The catalytic activity of bulk $\mathrm{Cu}$ after calcination is highly improved, resulting high furfural conversion of $79 \%$. When the calcination temperature was raised to $600{ }^{\circ} \mathrm{C}$, both $\mathrm{Cu}$ metal samples were totally oxidized into $\mathrm{CuO}$, which are inactive for the reaction. It is worth noting that it remains inactive for the furfural hydrogenation, when $\mathrm{CuO}$ was partially reduced into $\mathrm{Cu}(\mathrm{B} 5)$.

It is generally accepted that the $\mathrm{Cu} / \mathrm{Cu}^{0}$ heterojunction site played vital roles on furfural hydrogenation, which would be inactive when $\mathrm{Cu}^{\mathrm{I}}$ species was reduced into $\mathrm{Cu}^{0}$ species during catalysis. ${ }^{\mathrm{S} 12-\mathrm{S} 15}$ When both $\mathrm{Cu}$ samples were calcined at $300{ }^{\circ} \mathrm{C}$, part $\mathrm{Cu}$ metal was oxidized into $\mathrm{CuO}$ and $\mathrm{Cu}_{2} \mathrm{O}$ species to form $\mathrm{Cu} / \mathrm{Cu}_{2} \mathrm{O}$ heterojunction, which is favorable to the hydrogenation reaction (Table S9, entries 2 and 5). When $\mathrm{Cu}$ metal was totally oxidized into $\mathrm{CuO}$ at $600{ }^{\circ} \mathrm{C}$, both $\mathrm{Cu}$ metal samples showed no variation after reaction. Because the activation energy for direct reduction from $\mathrm{CuO}$ to $\mathrm{Cu}$ is lower than those to $\mathrm{Cu}_{2} \mathrm{O}$ and $\mathrm{Cu}_{4} \mathrm{O}_{3}$ intermediates, no $\mathrm{Cu}_{2} \mathrm{O}$ species appeared during reaction, which is unfavorable for the furfural hydrogenation, due to lack of $\mathrm{Cu}_{2} \mathrm{O}$ species. ${ }^{\mathrm{S} 16, \mathrm{~S} 17} \mathrm{It}$ is worth noting that nano $\mathrm{Cu}$ delivered $66 \%$ furfural conversion, because the edge of nano $\mathrm{Cu}$ particles could be oxidized into $\mathrm{Cu}_{2} \mathrm{O}$ species exposed in air. ${ }^{\mathrm{S} 18-\mathrm{S} 21}$ The above results indicate that the electronic variation on $\mathrm{Cu}$ surface is a key factor to improve the catalytic properties in the hydrogenation reaction. 


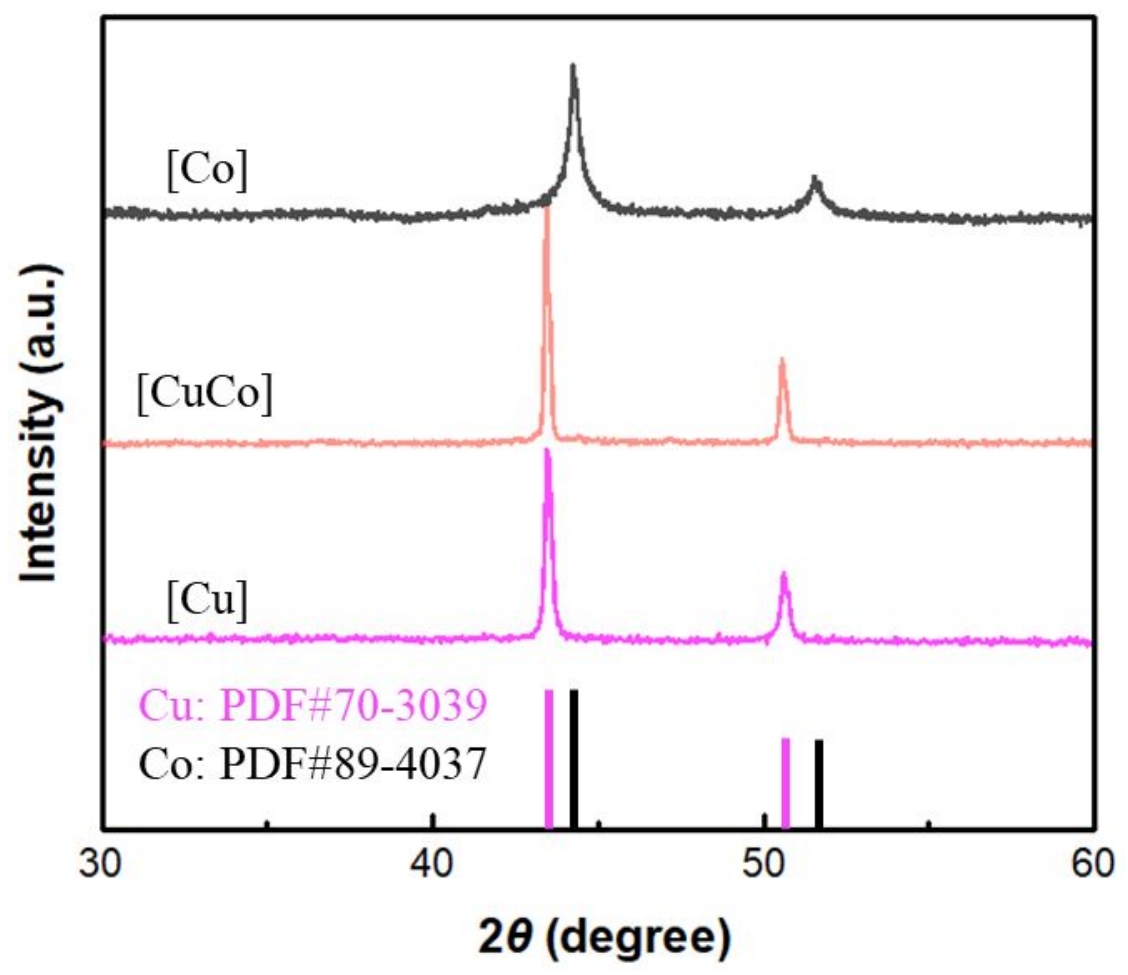

Figure S16. PXRD patterns for direct pyrolysis of $\mathrm{Cu}, \mathrm{Co}$ and $\mathrm{Cu} / \mathrm{Co}$ benzoate complexes at 700 ${ }^{\circ} \mathrm{C}$ in $\mathrm{Ar}$ atmosphere, denoted as $[\mathrm{Cu}],[\mathrm{Co}]$ and $[\mathrm{CuCo}]$, respectively. 


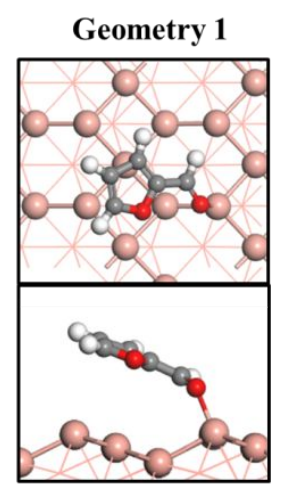

$E_{\text {ad }}=-42.69 \mathrm{~kJ} / \mathrm{mol}$

$d_{l(\mathrm{Cu}-\mathrm{O})}=1.99 \AA$

$d_{2(\mathrm{Cu}-\mathrm{O})}=3.99 \AA$

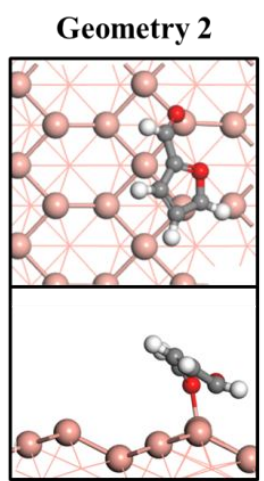

$E_{\text {ad }}=-49.20 \mathrm{~kJ} / \mathrm{mol}$

$d_{l(\mathrm{Cu}-\mathrm{O})}=2.02 \AA$

$d_{2(\mathrm{Cu}-\mathrm{O})}=2.73 \AA$

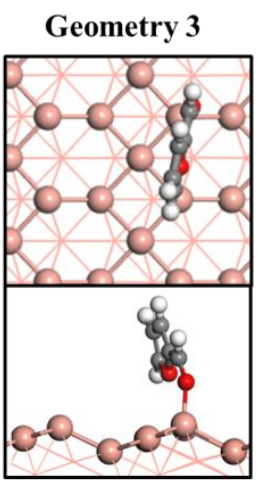

$E_{\text {ad }}=-42.46 \mathrm{~kJ} / \mathrm{mol}$

$d_{l(\mathrm{Cu}-\mathrm{O})}=1.99 \AA$

$d_{2(\mathrm{Cu}-\mathrm{O})}=2.63 \AA$
Geometry 4

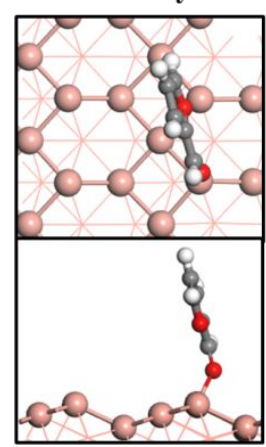

$E_{\text {ad }}=-33.85 \mathrm{~kJ} / \mathrm{mol}$

$d_{l(\mathrm{Cu}-\mathrm{O})}=1.96 \AA$

$d_{2(\mathrm{Cu}-\mathrm{O})}=3.82 \AA$
Geometry 5

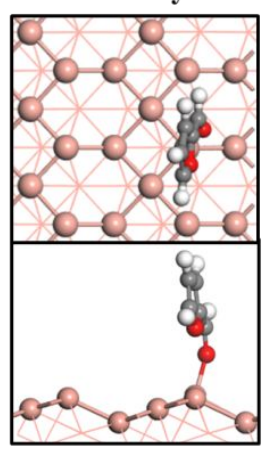

$E_{\text {ad }}=-52.35 \mathrm{~kJ} / \mathrm{mol}$

$d_{l(\mathrm{Cu}-\mathrm{O})}=1.99 \AA$

$d_{2(\mathrm{Cu}-\mathrm{O})}=3.46 \AA$

Figure S17. Optimized geometries for adsorption configurations of furfural on $\mathrm{Cu}(110)$ surface. The adsorption energies and $\mathrm{Cu}-\mathrm{O}$ distances are shown in text. 


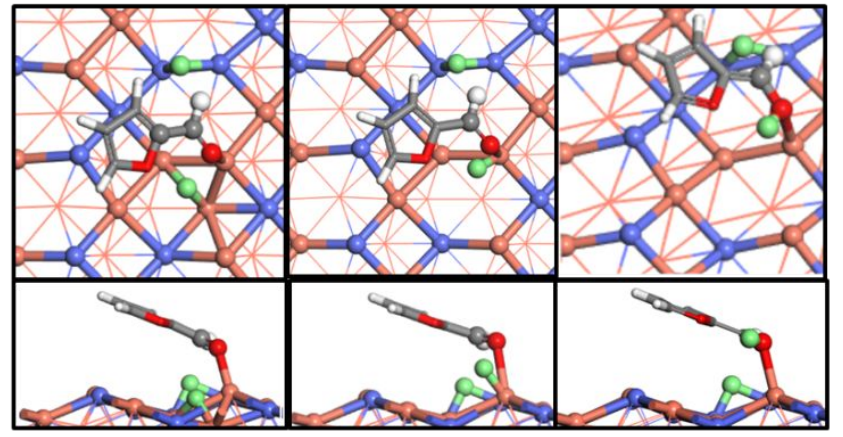

$$
\begin{array}{lll}
d_{(\mathrm{Cu}-\mathrm{H})}=1.82 \AA & d_{(\mathrm{Cu}-\mathrm{H})}=1.60 \AA & d_{(\mathrm{Cu}-\mathrm{H})}=2.65 \AA \\
d_{(\mathrm{O}-\mathrm{H})}=2.74 \AA d_{(\mathrm{O}-\mathrm{H})}=1.56 \AA & d_{(\mathrm{O}-\mathrm{H})}=0.98 \AA
\end{array}
$$

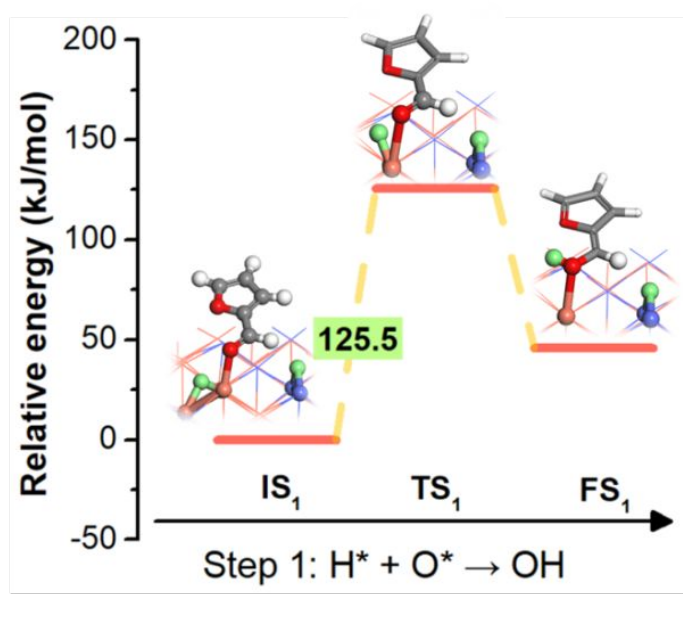

Figure S18. The structures of initial state, transition state and final state, and the potential energy diagram for furfural activation on $\mathrm{Cu}$ site of $\mathrm{Co}$-substituted $\mathrm{Cu}(110)$ surface. The highlight green balls represent $\mathrm{H}$ atoms. 

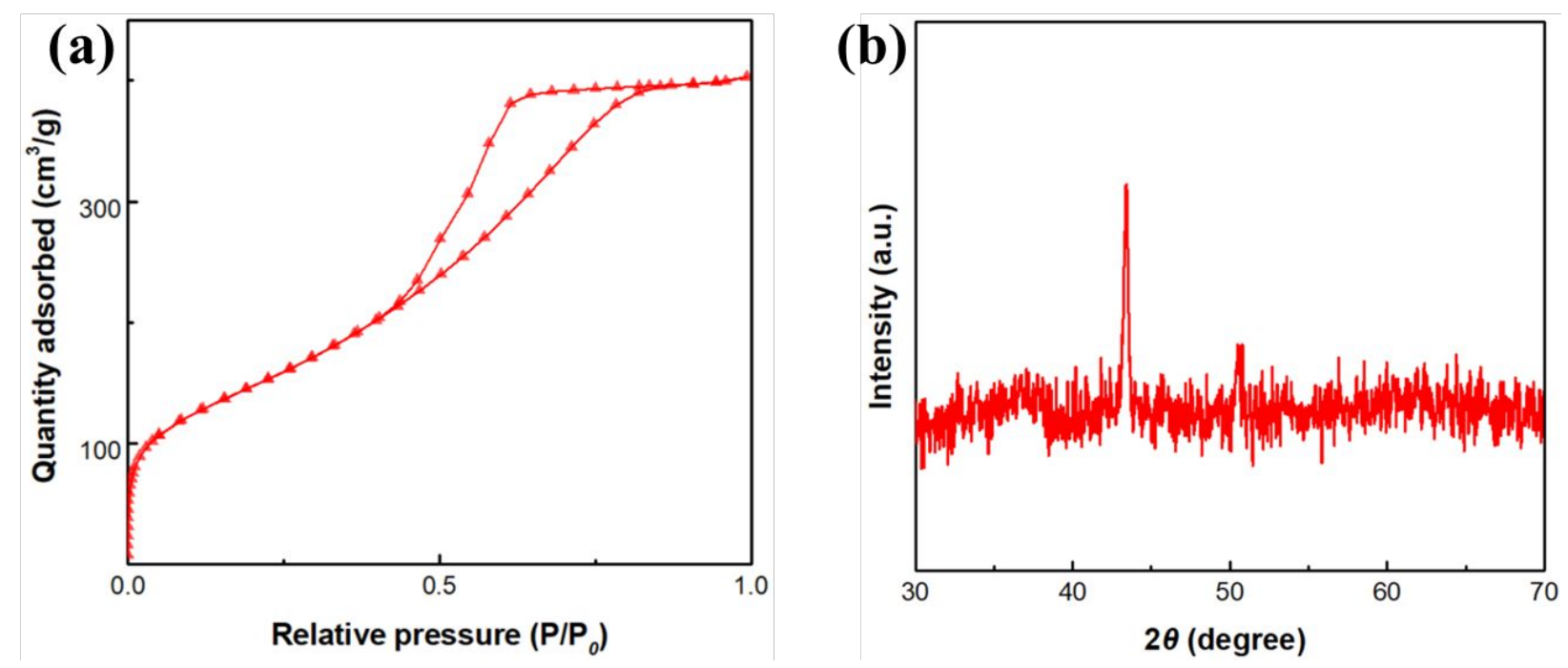

Figure S19. (a) $\mathrm{N}_{2}$ adsorption-desorption isotherms and (b) PXRD profile of recovered PMS-after catalysis. 


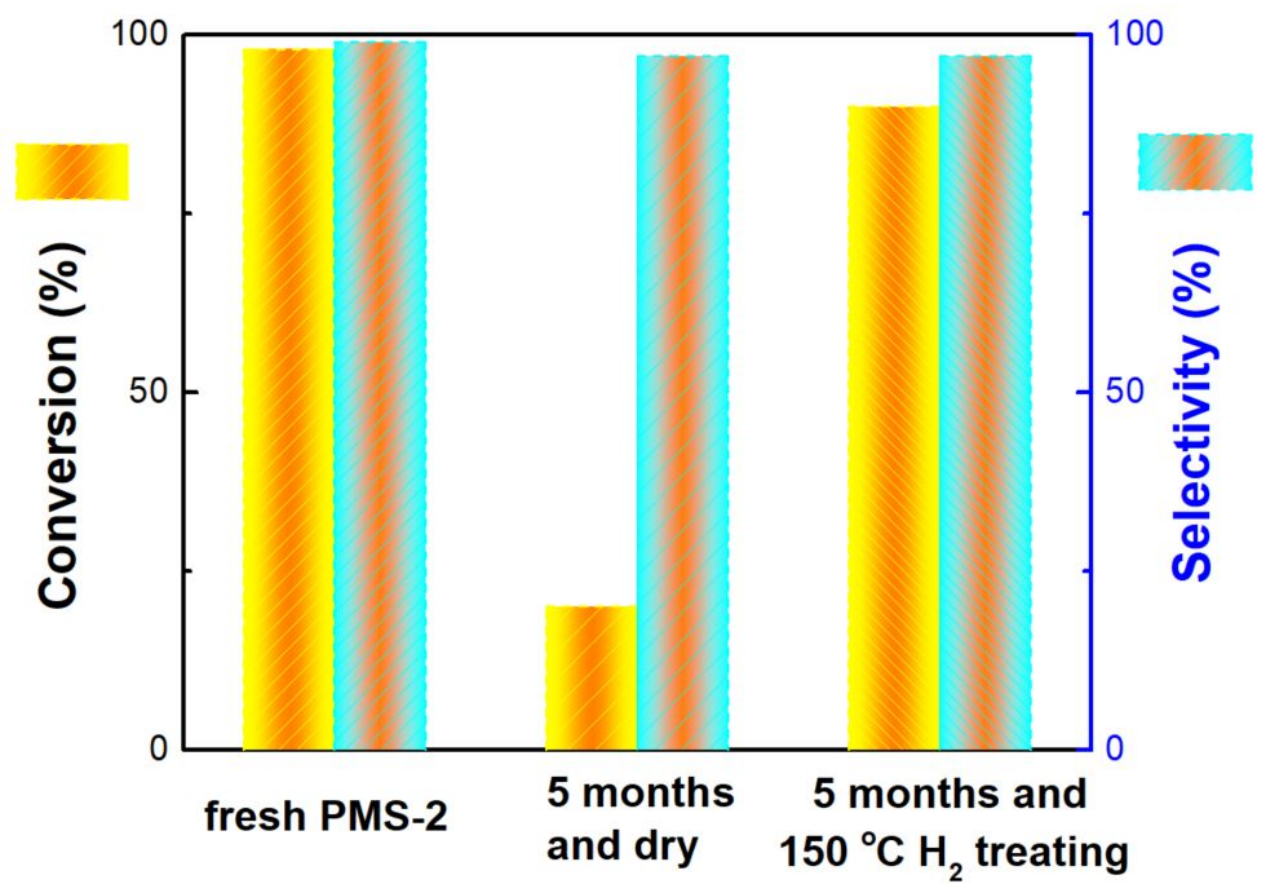

Figure S20. Stability study of PMS-2 for catalytic hydrogenation of furfural. Reaction conditions: $2.5 \mathrm{mmol}$ furfural, $0.1 \mathrm{mmol}(\mathrm{Cu}+\mathrm{Co})$ catalyst, $10 \mathrm{~mL}$ methanol, $1 \mathrm{MPa}_{2}, 130^{\circ} \mathrm{C}, 1 \mathrm{~h}$.

After being stored in air for five months, the conversion of furfural decreased from 79 to $29 \%$ over PMS-2, due to slow oxidation of the surficial metal species. The catalytic activity could be recovered in $\mathrm{H}_{2}$ flow at $150{ }^{\circ} \mathrm{C}$ for $1 \mathrm{~h}$, resulting highly improved furfural conversion of $90 \%$. 
Tables

Table S1. Textural properties of different PMS-2 precursors and PMS-2 materials ${ }^{[\mathrm{a}]}$

\begin{tabular}{lcccc}
\hline Material & $\begin{array}{c}\mathrm{N}_{2} \text { uptake } \\
\left(\mathrm{cm}^{3} \mathrm{~g}^{-1}\right)\end{array}$ & $\begin{array}{c}\mathrm{S}_{\mathrm{BET}} \\
\left(\mathrm{m}^{2} \mathrm{~g}^{-1}\right)\end{array}$ & $\begin{array}{c}\mathrm{V}_{\text {pore }} \\
\left(\mathrm{cm}^{3} \mathrm{~g}^{-1}\right)\end{array}$ & $\begin{array}{l}\mathrm{V}_{\text {micro }} \\
\left(\mathrm{cm}^{3} \mathrm{~g}^{-1}\right)\end{array}$ \\
\hline PMS-2 precursor $(\mathrm{Si}: \mathrm{M}=16)$ & 26 & 12 & 0.027 & $<0.001$ \\
PMS-2 $(\mathrm{Si}: \mathrm{M}=16)$ & 163 & 391 & 0.252 & 0.074 \\
PMS-2 precursor $(\mathrm{Si}: \mathrm{Cu}=4)$ & 8 & 18 & 0.047 & $<0.001$ \\
PMS-2 $(\mathrm{Si}: \mathrm{Cu}=4)$ & 352 & 675 & 0.775 & 0.001 \\
\hline
\end{tabular}

[a] Calculated from $\mathrm{N}_{2}$ physisorption isotherms. 
Table S2. Textual properties ${ }^{[a]}$ and elemental compositions ${ }^{[b]}$ for different PMS materials

\begin{tabular}{lccccccc}
\hline \multicolumn{1}{c}{ Sample } & $\begin{array}{c}\mathrm{S}_{\mathrm{BET}} \\
\left(\mathrm{m}^{2} \mathrm{~g}^{-1}\right)\end{array}$ & $\begin{array}{c}\mathrm{V}_{\text {pore }} \\
\left(\mathrm{cm}^{3} \mathrm{~g}^{-1}\right)\end{array}$ & $\begin{array}{c}\mathrm{V}_{\text {micro }} \\
\left(\mathrm{cm}^{3} \mathrm{~g}^{-1}\right)\end{array}$ & $\begin{array}{c}\mathrm{Cu} \\
\mathrm{wt} \%\end{array}$ & $\begin{array}{c}\mathrm{Co} \\
\mathrm{wt} \%\end{array}$ & $\begin{array}{c}\mathrm{Cu}+\mathrm{Co} \\
\mathrm{wt} \%\end{array}$ & $\begin{array}{c}\mathrm{Cu} / \mathrm{Co} \text { mole } \\
\text { ratio }\end{array}$ \\
\hline PMS-2-Cu & 642 & 0.563 & 0.008 & 17.3 & 0.0 & 17.3 & - \\
PMS-2-Cu $\mathrm{Co}$ & 516 & 0.643 & 0.003 & 13.5 & 4.4 & 17.9 & 2.8 \\
PMS-2 & 675 & 0.637 & 0.001 & 8.1 & 7.3 & 15.4 & 1.0 \\
PMS-2-CuCo 3 & 590 & 0.678 & 0.002 & 3.1 & 9.8 & 12.9 & 0.3 \\
PMS-2-Co & 535 & 0.873 & 0.018 & 0.0 & 15.6 & 15.6 & 0 \\
\hline
\end{tabular}

[a] Calculated from $\mathrm{N}_{2}$ physisorption isotherms. [b] Determined by ICP-OES. 
Table S3. Textual properties ${ }^{[a]}$ and catalytic hydrogen of furfural into furfuryl alcohol ${ }^{[b]}$ for different materials

\begin{tabular}{lllllllll}
\hline Sample & $\begin{array}{l}\text { XRD } \\
\text { peaks }\end{array}$ & $\begin{array}{l}\mathrm{S}_{\mathrm{BET}} \\
\left(\mathrm{m}^{2} \mathrm{~g}^{-1}\right)\end{array}$ & $\begin{array}{l}\mathrm{V}_{\text {pore }} \\
\left(\mathrm{cm}^{3} \mathrm{~g}^{-1}\right)\end{array}$ & $\begin{array}{l}\mathrm{V}_{\text {micro }} \\
\left(\mathrm{cm}^{3} \mathrm{~g}^{-1}\right)\end{array}$ & $\begin{array}{l}\text { Conv. } \\
(\%)\end{array}$ & $\begin{array}{l}\text { Sel. } \\
(\%)\end{array}$ & $\begin{array}{l}\text { Yield } \\
(\%)\end{array}$ & $\begin{array}{l}\text { TOF } \\
\left(\mathrm{h}^{-1}\right)\end{array}$ \\
\hline $\begin{array}{l}\text { PMS-2 } \\
(\mathrm{L}: \mathrm{M}=0: 1)\end{array}$ & $\mathrm{Cu} / \mathrm{Co}$ & 57 & 0.112 & 0.090 & 19 & 48 & 9 & 4.7 \\
$\begin{array}{l}\text { PMS-2 } \\
(\mathrm{L}: \mathrm{M}=4: 1)\end{array}$ & $\mathrm{Cu}$ & 675 & 0.775 & 0.001 & 99 & $>99$ & 99 & 24.9 \\
$\begin{array}{l}\text { PMS-2 } \\
(\mathrm{L}: \mathrm{M}=16: 1)\end{array}$ & $\mathrm{Cu} / \mathrm{Co}$ & 498 & 0.745 & 0.025 & 46 & $>99$ & 46 & 11.5 \\
\hline
\end{tabular}

[a] Calculated from $\mathrm{N}_{2}$ physisorption isotherms. [b] Reaction conditions: $2.5 \mathrm{mmol}$ furfural, 0.1 mmol $(\mathrm{Cu}+\mathrm{Co})$ catalyst, $10 \mathrm{~mL}$ methanol, $1 \mathrm{MPa}_{2}, 110^{\circ} \mathrm{C}, 1 \mathrm{~h}$. 
Table S4. Textual properties ${ }^{[a]}$ and catalytic hydrogen of furfural into furfuryl alcohol ${ }^{[b]}$ for PMS-2 annealed at different temperatures

\begin{tabular}{lllllllll}
\hline Material & $\begin{array}{l}\text { XRD } \\
\text { peaks }\end{array}$ & $\begin{array}{l}\mathrm{S}_{\mathrm{BET}} \\
\left(\mathrm{m}^{2} \mathrm{~g}^{-1}\right)\end{array}$ & $\begin{array}{l}\mathrm{V}_{\text {pore }} \\
\left(\mathrm{cm}^{3} \mathrm{~g}^{-1}\right)\end{array}$ & $\begin{array}{l}\mathrm{V}_{\text {micro }} \\
\left(\mathrm{cm}^{3} \mathrm{~g}^{-1}\right)\end{array}$ & $\begin{array}{l}\text { Conv. } \\
(\%)\end{array}$ & $\begin{array}{l}\text { Sel. } \\
(\%)\end{array}$ & $\begin{array}{l}\text { Yield } \\
(\%)\end{array}$ & $\begin{array}{l}\text { TOF } \\
\left(\mathrm{h}^{-1}\right)\end{array}$ \\
\hline $\begin{array}{l}\text { PMS-2 } \\
\left(\mathrm{Ar}-600^{\circ} \mathrm{C}\right)\end{array}$ & $\mathrm{Cu} / \mathrm{Co}$ & 695 & 0.795 & 0.003 & 28 & 86 & 24 & 7.0 \\
$\begin{array}{l}\text { PMS-2 } \\
\left(\mathrm{Ar}-700^{\circ} \mathrm{C}\right)\end{array}$ & $\mathrm{Cu}$ & 675 & 0.775 & 0.001 & 99 & $>99$ & 99 & 24.9 \\
$\mathrm{PMS}-2$ & $\mathrm{Cu}$ & 637 & 0.667 & 0.020 & 18 & 98 & 18 & 4.5 \\
$\left(\mathrm{Ar}-800{ }^{\circ} \mathrm{C}\right)$ & & & & & & & & \\
\hline
\end{tabular}

[a] Calculated from $\mathrm{N}_{2}$ physisorption isotherms. [b] Reaction conditions: $2.5 \mathrm{mmol}$ furfural, 0.1 mmol $(\mathrm{Cu}+\mathrm{Co})$ catalyst, $10 \mathrm{~mL}$ methanol, $1 \mathrm{MPa} \mathrm{H}_{2}, 110^{\circ} \mathrm{C}, 1 \mathrm{~h}$. 
Table S5. High resolution Cu 2p XPS data for different PMS materials

\begin{tabular}{|c|c|c|c|c|c|c|c|}
\hline \multirow{2}{*}{ Material } & \multicolumn{2}{|c|}{$\begin{array}{l}\text { Binding energy } \\
( \pm 0.1 \mathrm{eV})\end{array}$} & \multirow{2}{*}{$\begin{array}{c}\text { Splitting } \\
(\mathrm{eV})\end{array}$} & \multicolumn{2}{|c|}{$\begin{array}{l}\text { Binding energy } \\
( \pm 0.1 \mathrm{eV})\end{array}$} & \multirow{2}{*}{$\begin{array}{c}\text { Splitting } \\
(\mathrm{eV})\end{array}$} & \multirow{2}{*}{$\begin{array}{c}\mathrm{Cu}^{0} / \mathrm{Cu}^{2-} \\
\text { ratio }\end{array}$} \\
\hline & $\begin{array}{c}\mathrm{Cu}^{0 / 1+} \\
2 p_{3 / 2} \\
\end{array}$ & $\begin{array}{c}\mathrm{Cu}^{0 / 1+} \\
2 p_{1 / 2} \\
\end{array}$ & & $\begin{array}{l}\mathrm{Cu}^{2+} \\
2 p_{3 / 2}\end{array}$ & $\begin{array}{l}\mathrm{Cu}^{2+} \\
2 p_{1 / 2}\end{array}$ & & \\
\hline PMS-2-Cu & 932.4 & 952.3 & 19.9 & 934.8 & 954.1 & 19.3 & $1: 0.21$ \\
\hline PMS-2- $\mathrm{Cu}_{3} \mathrm{Co}$ & 932.4 & 952.1 & 19.7 & 935.1 & 954.5 & 19.4 & $1: 0.15$ \\
\hline PMS-2 & 932.4 & 952.1 & 19.7 & 935.5 & 955.0 & 19.5 & $1: 0.19$ \\
\hline PMS-2-CuCo 3 & 932.1 & 952.0 & 19.9 & 935.6 & 955.3 & 19.7 & $1: 0.20$ \\
\hline PMS-2-Co & - & - & - & - & - & - & - \\
\hline
\end{tabular}

The data are related to Figure S13. 
Table S6. High resolution Co 2p XPS data for different PMS materials

\begin{tabular}{|c|c|c|c|c|c|c|c|}
\hline \multirow{2}{*}{ Material } & \multicolumn{2}{|c|}{$\begin{array}{l}\text { Binding energy } \\
( \pm 0.1 \mathrm{eV})\end{array}$} & \multirow{2}{*}{$\begin{array}{l}\text { Splitting } \\
(\mathrm{eV})\end{array}$} & \multicolumn{2}{|c|}{$\begin{array}{l}\text { Binding energy } \\
\quad( \pm 0.1 \mathrm{eV})\end{array}$} & \multirow{2}{*}{$\begin{array}{l}\text { Splitting } \\
(\mathrm{eV})\end{array}$} & \multirow{2}{*}{$\begin{array}{c}\mathrm{Co}^{0} / \mathrm{Co}^{2+} \\
\text { ratio }\end{array}$} \\
\hline & $\begin{array}{c}\mathrm{Co}^{0} \\
2 p_{3 / 2} \\
\end{array}$ & $\begin{array}{c}\mathrm{Co}^{0} \\
2 p_{1 / 2}\end{array}$ & & $\begin{array}{l}\mathrm{Co}^{2+} \\
2 p_{3 / 2}\end{array}$ & $\begin{array}{l}\mathrm{Co}^{2+} \\
2 p_{1 / 2}\end{array}$ & & \\
\hline PMS-2-Cu & - & - & - & - & - & - & - \\
\hline PMS-2- $\mathrm{Cu}_{3} \mathrm{Co}$ & 778.5 & 793.3 & 14.8 & 781.8 & 797.7 & 15.9 & $1: 0.25$ \\
\hline PMS-2 & 778.5 & 793.5 & 15.1 & 781.7 & 797.6 & 16.0 & $1: 0.24$ \\
\hline PMS-2-CuCo 3 & 778.5 & 793.4 & 14.9 & 781.9 & 797.7 & 15.8 & $1: 0.25$ \\
\hline PMS-2-Co & 778.4 & 793.4 & 15.0 & 781.9 & 797.8 & 15.9 & $1: 0.28$ \\
\hline
\end{tabular}

The data are related to Figure S14. 
Table S7. Convergence tests with various parameters of layer thickness and $k$-points

\begin{tabular}{cccccc}
\hline Entry & $\begin{array}{c}\text { Layer } \\
\text { numbers }\end{array}$ & $k$-points & $\begin{array}{c}\text { Energy cutoff } \\
(\mathrm{eV})\end{array}$ & $\begin{array}{c}\text { Formation energy } \\
(\mathrm{eV})\end{array}$ & $\begin{array}{c}\text { Surface energy } \\
\left(\gamma \mathrm{J} / \mathrm{m}^{2}\right)\end{array}$ \\
\hline 1 & three & $5 \times 5 \times 1$ & 450 & 23.52 & 1.05 \\
2 & four & $5 \times 5 \times 1$ & 450 & 37.35 & 1.66 \\
3 & five & $5 \times 5 \times 1$ & 450 & 24.63 & 1.09 \\
4 & six & $5 \times 5 \times 1$ & 450 & 24.65 & 1.09 \\
5 & seven & $5 \times 5 \times 1$ & 450 & 24.62 & 1.09 \\
6 & six & $1 \times 1 \times 1$ & 450 & 26.73 & 1.19 \\
7 & six & $2 \times 2 \times 1$ & 450 & 25.04 & 1.11 \\
8 & six & $3 \times 3 \times 1$ & 450 & 24.65 & 1.10 \\
9 & six & $4 \times 4 \times 1$ & 450 & 24.59 & 1.09 \\
10 & six & $6 \times 6 \times 1$ & 450 & 24.57 & 1.09 \\
11 & six & $5 \times 5 \times 1$ & 300 & 24.38 & 1.08 \\
12 & six & $5 \times 5 \times 1$ & 350 & 24.38 & 1.08 \\
13 & six & $5 \times 5 \times 1$ & 400 & 24.51 & 1.09 \\
14 & six & $5 \times 5 \times 1$ & 500 & 24.65 & 1.10 \\
15 & six & $5 \times 5 \times 1$ & 550 & 24.65 & 1.10 \\
\hline
\end{tabular}

The Monkhorst-Pack $k$-points, layers and energy cutoff in the reciprocal lattice are sufficient to guarantee convergence in formation energy and surface energy. 
Table S8. Spatial distortion resulted from substitution of $\mathrm{Cu}$ atoms on the $\mathrm{Cu}(110)$ surface by $\mathrm{Co}$ atoms

\begin{tabular}{ccccc}
\hline Co number & $x$ axis (\%) & $y$ axis (\%) & $z$ axis (\%) & $\triangle d(\AA)$ \\
\hline Co-1 & 0.01 & 0.03 & 0.01 & 0.05 \\
Co-2 & 0.04 & 0.05 & 0.04 & 0.12 \\
Co-3 & 0.00 & 0.01 & 0.00 & 0.01 \\
Co-4 & 0.00 & 0.05 & 0.00 & 0.07 \\
Co-5 & 0.00 & 0.03 & 0.00 & 0.04 \\
Co-6 & 0.02 & 0.03 & 0.00 & 0.04 \\
Co-7 & 0.00 & 0.02 & 0.01 & 0.04 \\
Co-8 & 0.01 & 0.01 & 0.00 & 0.02 \\
Co-9 & 0.01 & 0.03 & 0.00 & 0.04 \\
\hline
\end{tabular}


Table S9. Catalytic hydrogen of furfural into furfuryl alcohol and phase changes for nano $\mathrm{Cu}$ and bulk $\mathrm{Cu}^{[\mathrm{a}]}$

\begin{tabular}{ccccc}
\hline \multirow{2}{*}{ Entry } & Catalyst & Conv. $(\%)$ & \multicolumn{2}{c}{ Crystal phase } \\
\cline { 3 - 5 } & raw nano $\mathrm{Cu}^{[\mathrm{b}]}$ & 66 & $\mathrm{Cu}$ & $\mathrm{Cu}$ \\
\hline 1 & nano $\mathrm{Cu}+$ Air $300^{\circ} \mathrm{C}^{[\mathrm{d}]}$ & 51 & $\mathrm{Cu} / \mathrm{Cu}_{2} \mathrm{O} / \mathrm{CuO}$ & $\mathrm{Cu}$ \\
2 & nano $\mathrm{Cu}+$ Air $600^{\circ} \mathrm{C}^{[\mathrm{d}]}$ & 0 & $\mathrm{CuO}$ & $\mathrm{CuO}$ \\
4 & raw bulk $\mathrm{Cu}^{[\mathrm{c}]}$ & 0 & $\mathrm{Cu}$ & $\mathrm{Cu}$ \\
5 & bulk Cu + Air $300{ }^{\circ} \mathrm{C}^{[\mathrm{d}]}$ & 79 & $\mathrm{Cu} / \mathrm{Cu}_{2} \mathrm{O} / \mathrm{CuO}$ & $\mathrm{Cu}$ \\
6 & bulk Cu + Air $600{ }^{\circ} \mathrm{C}^{[\mathrm{d}]}$ & 0 & $\mathrm{CuO}$ & $\mathrm{Cu} / \mathrm{CuO}$ \\
\hline
\end{tabular}

[a] Reaction conditions: $1.0 \mathrm{mmol}$ furfural, $1.5 \mathrm{mmol} \mathrm{Cu}$ catalyst, $10 \mathrm{~mL}$ methanol, $1 \mathrm{MPa} \mathrm{H}_{2}, 140$ ${ }^{\circ} \mathrm{C}, 6 \mathrm{~h}$. [b] The labeled particle size is $60-100 \mathrm{~nm}$. [c] Particle size is at micron scale. [d] The samples were treated at specified temperature for $1 \mathrm{~h}$ with a ramp rate of $5^{\circ} \mathrm{C} / \mathrm{min}$. 
Table S10. Representative work for liquid-phase hydrogenation of furfural into furfuryl alcohol in the literature

\begin{tabular}{|c|c|c|c|c|c|}
\hline Material & Reaction conditions & $\begin{array}{c}\text { Conv. } \\
(\%)\end{array}$ & $\begin{array}{l}\text { Sel. } \\
(\%)\end{array}$ & $\begin{array}{l}\text { TOF } \\
\left(h^{-1}\right)\end{array}$ & $\begin{array}{l}\text { Year \& } \\
\text { Ref. }\end{array}$ \\
\hline $\mathrm{Cu}-\mathrm{Cr}$ & $\begin{array}{l}200^{\circ} \mathrm{C}, 4 \mathrm{~h}, 60 \mathrm{bar}, 2.1 \mathrm{~mL} \text { furfural, } 0.2 \\
\mathrm{~g} \text { catalyst, octane }\end{array}$ & $>99$ & 95 & 0.6 & $2013^{\mathrm{S} 22}$ \\
\hline $\mathrm{CuMgAl}$ & $\begin{array}{l}110^{\circ} \mathrm{C}, 4 \mathrm{~h}, 1 \mathrm{MPa} \mathrm{H}_{2}, 6 \mathrm{mmol} \text { furfural, } \\
0.1 \mathrm{~g} \text { catalyst, 2-propanol }\end{array}$ & $>99$ & 66 & 2.9 & $2013^{\mathrm{S} 23}$ \\
\hline $\mathrm{Cu}_{40} \mathrm{MgAl}$ & $\begin{array}{l}150^{\circ} \mathrm{C}, 4 \mathrm{~h}, 1 \mathrm{MPa} \mathrm{H}_{2}, 6 \mathrm{mmol} \text { furfural, } \\
0.2 \mathrm{~g} \text { catalyst, 2-propanol }\end{array}$ & $>99$ & $>99$ & 1.6 & $2015^{\mathrm{S} 24}$ \\
\hline $\mathrm{Co} / \mathrm{SBA}-15$ & $\begin{array}{l}150^{\circ} \mathrm{C}, 2 \mathrm{~h}, 2 \mathrm{MPa} \mathrm{H}_{2}, \\
\text { furfural: catalyst }=2: 1 \text {, water }\end{array}$ & $>99$ & 76 & 46 & $2015^{\mathrm{s} 25}$ \\
\hline $\mathrm{Cu}-\mathrm{Fe}$ & $\begin{array}{l}160{ }^{\circ} \mathrm{C}, 5 \mathrm{~h}, 9 \mathrm{MPa} \mathrm{H}_{2}, 25 \mathrm{mmol} \\
\text { furfural, } 0.2 \mathrm{~g} \text { catalyst, 2-propanol }\end{array}$ & 99 & 91 & 1.8 & $2015^{\mathrm{S} 26}$ \\
\hline $\mathrm{Cu}-\mathrm{Co} / \mathrm{SiO}_{2}$ & $\begin{array}{l}200^{\circ} \mathrm{C}, 4 \mathrm{~h}, 4 \mathrm{MPa} \mathrm{H}_{2}, 1.0 \mathrm{~g} \text { catalyst, } \\
44.7 \mathrm{mmol} \text { furfural, ethanol }\end{array}$ & 85 & 62 & 2.4 & $2016^{\mathrm{S} 27}$ \\
\hline $\mathrm{CuCo}_{0.5} / \mathrm{C}$ & $\begin{array}{l}140{ }^{\circ} \mathrm{C}, 1 \mathrm{~h}, 3 \mathrm{MPa} \mathrm{H}_{2}, 0.7 \mathrm{mmol} \\
\text { catalyst, } 15 \mathrm{wt} \% \text { furfural in ethanol }\end{array}$ & 99 & 97 & - & $2017^{\mathrm{S} 28}$ \\
\hline $\begin{array}{l}\mathrm{Cu} / \mathrm{AC}- \\
\mathrm{SOO}_{3} \mathrm{H}\end{array}$ & $\begin{array}{l}105^{\circ} \mathrm{C}, 3 \mathrm{~h}, 0.4 \mathrm{MPa} \mathrm{H}_{2}, \\
\text { catalyst: furfural = 1:1, 2-propanol }\end{array}$ & $>99$ & $>99$ & 5.4 & $2017^{\mathrm{S} 14}$ \\
\hline $\mathrm{CuCo}_{0.5} @ \mathrm{C}$ & $\begin{array}{l}150{ }^{\circ} \mathrm{C}, 3 \mathrm{~h}, 0.5 \mathrm{MPa} \mathrm{H}_{2}, 20 \mathrm{mg} \\
\text { catalyst, } 0.6 \mathrm{mmol} \text { furfural, water }\end{array}$ & $>99$ & 60 & 7.8 & $2018^{\mathrm{S} 29}$ \\
\hline $\mathrm{Ni}_{3} \mathrm{Fe}_{1} / \mathrm{SiO}_{2}$ & $\begin{array}{l}140{ }^{\circ} \mathrm{C}, 150 \mathrm{~min}, 3.4 \mathrm{MPa}_{2}, 1.2 \mathrm{~g} \\
\text { catalyst, } 2.5 \mathrm{mmol} \text { furfural, methanol }\end{array}$ & 99 & 97 & - & $2018^{\mathrm{S} 30}$ \\
\hline $\mathrm{Ni} / \mathrm{Al}_{2} \mathrm{O}_{3}-\mathrm{C}$ & $\begin{array}{l}160^{\circ} \mathrm{C}, 6 \mathrm{~h}, 4 \mathrm{MPa}_{2}, 0.6 \mathrm{~g} \text { catalyst, } 4 \\
\mathrm{~mL} \text { furfural, ethanol }\end{array}$ & 99 & 93 & 4.9 & $2019^{\mathrm{S} 31}$ \\
\hline $\mathrm{Cu}_{0.4} \mathrm{Mg}_{5.6} \mathrm{Al}_{2}$ & $\begin{array}{l}110^{\circ} \mathrm{C}, 2 \mathrm{~h}, 2 \mathrm{MPa} \mathrm{H}_{2}, 64 \mathrm{mg} \text { catalyst, } \\
1.0 \mathrm{mmol} \text { furfural, water }\end{array}$ & 99 & 99 & - & $2019^{\mathrm{S} 32}$ \\
\hline $\begin{array}{l}\mathrm{ZJU}-199-350 \\
\left(\mathrm{Cu} / \mathrm{Cu}_{2} \mathrm{O}\right)\end{array}$ & $\begin{array}{l}130^{\circ} \mathrm{C}, 3 \mathrm{~h}, 1 \mathrm{MPa} \mathrm{H}_{2}, 5.3 \mathrm{mg} \text { catalyst, } \\
1.0 \mathrm{mmol} \mathrm{FAL}, 2 \text {-propanol }\end{array}$ & 97 & 97 & 13.0 & $2020^{\mathrm{S} 12}$ \\
\hline PMS-2 & $\begin{array}{l}50{ }^{\circ} \mathrm{C}, 4 \mathrm{~h}, 1 \mathrm{MPa} \mathrm{H}_{2}, 0.5 \mathrm{mmol} \\
\text { catalyst, } 2.5 \mathrm{mmol} \mathrm{FAL} \text {, methanol }\end{array}$ & 99 & $>99$ & 1.2 & $\begin{array}{l}\text { This } \\
\text { work }\end{array}$ \\
\hline PMS-2 & $\begin{array}{l}110{ }^{\circ} \mathrm{C}, 1 \mathrm{~h}, 1 \mathrm{MPa} \mathrm{H}_{2}, 0.1 \mathrm{mmol} \\
\text { catalyst, } 2.5 \mathrm{mmol} \mathrm{FAL} \text {, methanol }\end{array}$ & 99 & $>99$ & 24.9 & $\begin{array}{l}\text { This } \\
\text { work }\end{array}$ \\
\hline
\end{tabular}


Table S11. Hydrogenation of furfural catalyzed by PMS-2-Cu, PMS-2 and PMS-2-Co at various temperatures

\begin{tabular}{|c|c|c|c|c|c|}
\hline Catalyst & Temperature $\left({ }^{\circ} \mathrm{C}\right)$ & Conversion $(\%)$ & Selectivity (\%) & Yield $(\%)$ & TOF $\left(\mathrm{h}^{-1}\right)$ \\
\hline \multirow{5}{*}{ PMS-2-Cu } & 80 & 0 & - & 0 & 0 \\
\hline & 90 & 13 & $>99$ & 13 & 3.2 \\
\hline & 100 & 34 & $>99$ & 34 & 8.5 \\
\hline & 110 & 55 & $>99$ & 55 & 13.7 \\
\hline & 120 & 87 & 94 & 81 & 21.7 \\
\hline \multirow{7}{*}{ PMS-2 } & 50 & 6 & $>99$ & 6 & 1.5 \\
\hline & 60 & 14 & $>99$ & 14 & 3.5 \\
\hline & 70 & 22 & $>99$ & 22 & 5.5 \\
\hline & 80 & 33 & $>99$ & 33 & 8.2 \\
\hline & 90 & 47 & $>99$ & 47 & 11.8 \\
\hline & 100 & 74 & $>99$ & 74 & 18.5 \\
\hline & 110 & 99 & $>99$ & 99 & 24.9 \\
\hline \multirow{5}{*}{ PMS-2-Co } & 80 & 0 & - & 0 & 0 \\
\hline & 90 & 2 & $>99$ & 2 & 0.5 \\
\hline & 100 & 4 & $>99$ & 4 & 1.0 \\
\hline & 110 & 8 & 98 & 7 & 2.0 \\
\hline & 120 & 17 & 98 & 17 & 4.2 \\
\hline
\end{tabular}

Reaction conditions: $2.5 \mathrm{mmol}$ furfural, $0.1 \mathrm{mmol}(\mathrm{Cu}+\mathrm{Co})$ catalyst, $10 \mathrm{~mL}$ methanol, $1 \mathrm{MPa} \mathrm{H}_{2}$, $1 \mathrm{~h}$. 
Table S12. Hydrogenation of furfural into furfuryl alcohol over various catalysts ${ }^{[a]}$

\begin{tabular}{ccccccc}
\hline Entry & Catalyst & T $\left({ }^{\circ} \mathrm{C}\right)$ & Conv. $(\%)$ & Sel. $(\%)$ & TOF $\left(\mathrm{h}^{-1}\right)$ & Mass Balance $^{[\mathrm{b}]}$ \\
\hline 1 & {$[\mathrm{Cu}]$} & 110 & 0 & - & - & - \\
2 & {$[\mathrm{CuCo}]$} & 110 & 3 & $>99$ & 0.8 & 97 \\
3 & {$[\mathrm{Co}]$} & 110 & 0 & - & - & - \\
\hline
\end{tabular}

[a] Reaction conditions: $2.5 \mathrm{mmol}$ furfural, $0.1 \mathrm{mmol}(\mathrm{Cu}+\mathrm{Co})$ catalyst, $10 \mathrm{~mL}$ methanol, $1 \mathrm{MPa}$ $\mathrm{H}_{2}, 1 \mathrm{~h}$. 
Table S13. Atomic valance state on pure $\mathrm{Cu}$ (110) surface and Co-substituted $\mathrm{Cu}$ (110) surface before/after furfural adsorption

\begin{tabular}{|c|c|c|c|c|c|c|}
\hline \multirow[t]{2}{*}{ Atom } & \multicolumn{3}{|c|}{ Pure $\mathrm{Cu}(110)$ surface } & \multicolumn{3}{|c|}{ Co-substituted $\mathrm{Cu}(110)$ surface } \\
\hline & unabsorbed & adsorbed & $\triangle$ & unabsorbed & adsorbed & $\triangle$ \\
\hline metal & $0.02^{+}$ & $0.31^{+}$ & +0.31 & $0.08^{+}$ & $0.33+$ & +0.25 \\
\hline $\mathrm{H}(-$ metal $)$ & $0.00^{+}$ & $0.23^{-}$ & -0.23 & $0.00^{+}$ & $0.23^{-}$ & -0.23 \\
\hline $\mathrm{C}(=\mathrm{O})$ & $0.92^{+}$ & $0.86^{+}$ & -0.06 & $0.92^{+}$ & $0.92^{+}$ & 0.00 \\
\hline $\mathrm{O}(=\mathrm{C})$ & $0.99^{-}$ & $1.09^{-}$ & -0.10 & $0.99^{-}$ & $1.06^{-}$ & -0.07 \\
\hline $\mathrm{H}(-\mathrm{C})$ & $0.05^{+}$ & $0.04^{+}$ & -0.01 & $0.05^{+}$ & $0.09^{+}$ & +0.04 \\
\hline
\end{tabular}




\section{References}

(S1) Kresse, G.; Furthmüller, J. Efficient Iterative Schemes for Ab Initio Total-Energy Calculations Using a Plane-Wave Basis Set. Phys. Rev. B 1996, 54, 11169-11186.

(S2) Kresse, G.; Furthmüller, J. Efficiency of Ab Initio Total Energy Calculations for Metals and Semiconductors Using a Plane Wave Basis Set. Comput. Mat. Sci. 1996, 6, 15-50.

(S3) Chen, M.; Yang, X. B.; Cui, J.; Tang, J. J.; Gan, L. Y.; Zhu, M.; Zhao, Y. J. Stability of Transition Metals on $\operatorname{Mg}(0001)$ Surfaces and Their Effects on Hydrogen Adsorption. Int. J. Hydrogen Energy. 2012, 37, 309-317.

(S4) Monkhorst, H. J.; Pack, J. D. Special Points for Brillouin-Zon Integrations. Phys. Rev. B 1976, 13, 5188-5192.

(S5) Henkelman, G.; Jónsson, H. Improved Tangent Estimate in the Nudged Elastic Band Method for Finding Minimum Energy Paths and Saddle Points. J. Chem. Phys. 2000, 113, 99789985.

(S6) (a) Henkelman, G.; Arnaldsson, A.; Jónsson, H. A Fast and Robust Algorithm for Bader Decomposition of Charge Density. Comput. Mater. Sci. 2006, 36, 354-360.

(S7) Sanville, E.; Kenny, S. D.; Smith, R.; Henkelman, G. Improved Grid-Based Algorithm for Bader Charge Allocation. J. Comput. Chem. 2007, 28, 899-908.

(S8) Park, S.; Kannapu, H. P. R.; Jeong, C.; Kim, J.; Suh, Y. W. Highly Active Mesoporous $\mathrm{Cu}-\mathrm{Al}_{2} \mathrm{O}_{3}$ Catalyst for the Hydrodeoxygenation of Furfural to 2-Methylfuran. ChemCatChem 2020, 12, 105-111.

(S9) Espinós, J. P.; Morales, J.; Barranco, A.; Caballero, A.; Holgado, J. P.; González-Elipe, A. R. Interface Effects for $\mathrm{Cu}, \mathrm{CuO}$, and $\mathrm{Cu}_{2} \mathrm{O}$ Deposited on $\mathrm{SiO}_{2}$ and $\mathrm{ZrO}_{2}$. XPS Determination of the Valence State of Copper in $\mathrm{Cu} / \mathrm{SiO}_{2}$ and $\mathrm{Cu} / \mathrm{ZrO}_{2}$ Catalysts. J. Phys. Chem. B 2002, 106, 6921-6929. 
(S10) Castner, D. G.; Watson, P. R.; Chan, I. Y. X-Ray Absorption Spectroscopy, X-Ray Photoelectron Spectroscopy, and Analytical Electron Microscopy Studies of Cobalt Catalysts. 2. Hydrogen Reduction Properties. J. Phys. Chem. 1990, 94, 819-828.

(S11) Chen, H.; Shen, K.; Tan, Y.; Li, Y. Multishell Hollow Metal/Nitrogen/Carbon Dodecahedrons with Precisely Controlled Architectures and Synergistically Enhanced Catalytic Properties. ACS Nano 2019, 13, 7800-7810.

(S12) Chen, K.; Ling, J.-L.; Wu, C.-D. In Situ Generation and Stabilization of Accessible $\mathrm{Cu} / \mathrm{Cu}_{2} \mathrm{O}$ Heterojunctions inside Organic Frameworks for Highly Efficient Catalysis. Angew. Chem. Int. Ed. 2020, 59, 1925-1931.

(S13) Liu, D.; Zemlyanov, D.; Wu, T.; Lobo-Lapidus, R. J.; Dumesic, J. A.; Miller, J. T.; Marshall, C. L. Deactivation Mechanistic Studies of Copper Chromite Catalyst for Selective Hydrogenation of 2-Furfuraldehyde. J. Catal. 2013, 299, 336-345.

(S14) Gong, W.; Chen, C.; Zhang, Y.; Zhou, H.; Wang, H.; Zhang, H.; Zhang, Y.; Wang, G.; Zhao, H. Efficient Synthesis of Furfuryl Alcohol from $\mathrm{H}_{2}$-Hydrogenation/Transfer Hydrogenation of Furfural Using Sulfonate Group Modified Cu Catalyst. ACS Sustain. Chem. Eng. 2017, 5, 2172 2180.

(S15) Rao, R. S.; Baker, R. T. K.; Vannice, M. A. Furfural Hydrogenation over CarbonSupported Copper. Catal. Letters 1999, 60, 51-57.

(S16) Rodriguez, J. A.; Kim, J. Y.; Hanson, J. C.; Pérez, M.; Frenkel, A. I. Reduction of CuO in $\mathrm{H}_{2}$ : In Situ Time-Resolved XRD Studies. Catal. Letters 2003, 85, 247-254.

(S17) Wang, X.; Hanson, J. C.; Frenkel, A. I.; Kim, J. Y.; Rodriguez, J. A. Time-Resolved Studies for the Mechanism of Reduction of Copper Oxides with Carbon Monoxide: Complex Behavior of Lattice Oxygen and the Formation of Suboxides. J. Phys. Chem. B 2004, 108, 1366713673. 
(S18) Reske, R.; Mistry, H.; Behafarid, F.; Roldan Cuenya, B.; Strasser, P. Particle Size Effects in the Catalytic Electroreduction of $\mathrm{CO}_{2}$ on Cu Nanoparticles. J. Am. Chem. Soc. 2014, $136,6978-6986$.

(S19) Sun, C.; Zeng, P.; He, M.; He, X.; Xie, X. Morphological Effect of Non-Supported Copper Nanocrystals on Furfural Hydrogenation. Catal. Commun. 2016, 86, 5-8.

(S20) Li, X.; Jia, P.; Wang, T. Furfural: A Promising Platform Compound for Sustainable Production of C4 and C5 Chemicals. ACS Catal. 2016, 6, 7621-7640.

(S21) Xia, Y.; Xia, X.; Peng, H. C. Shape-Controlled Synthesis of Colloidal Metal Nanocrystals: Thermodynamic versus Kinetic Products. J. Am. Chem. Soc. 2015, 137, 7947-7966.

(S22) Yan, K.; Chen, A. Efficient Hydrogenation of Biomass-Derived Furfural and Levulinic Acid on the Facilely Synthesized Noble-Metal-Free Cu-Cr Catalyst. Energy 2013, 58, 357-363.

(S23) Villaverde, M. M.; Bertero, N. M.; Garetto, T. F.; Marchi, A. J. Selective Liquid-Phase Hydrogenation of Furfural to Furfuryl Alcohol over Cu-Based Catalysts. Catal. Today 2013, 213, $87-92$.

(S24) Villaverde, M. M.; Garetto, T. F.; Marchi, A. J. Liquid-Phase Transfer Hydrogenation of Furfural to Furfuryl Alcohol on Cu-Mg-Al Catalysts. Catal. Commun. 2015, 58, 6-10.

(S25) Audemar, M.; Ciotonea, C.; De Oliveira Vigier, K.; Royer, S.; Ungureanu, A.; Dragoi, B.; Dumitriu, E.; Jérôme, F. Selective Hydrogenation of Furfural to Furfuryl Alcohol in the Presence of a Recyclable Cobalt/SBA-15 Catalyst. ChemSusChem 2015, 8, 1885-1891.

(S26) Yan, K.; Chen, A. Selective Hydrogenation of Furfural and Levulinic Acid to Biofuels on the Ecofriendly Cu-Fe Catalyst. Fuel 2014, 115, 101-108.

(S27) Srivastava, S.; Jadeja, G. C.; Parikh, J. A Versatile Bi-Metallic Copper-Cobalt Catalyst for Liquid Phase Hydrogenation of Furfural to 2-Methylfuran. RSC Adv. 2016, 6, 1649-1658. 
(S28) Wang, Y.; Miao, Y.; Li, S.; Gao, L.; Xiao, G. Metal-Organic Frameworks Derived Bimetallic Cu-Co Catalyst for Efficient and Selective Hydrogenation of Biomass-Derived Furfural to Furfuryl Alcohol. Mol. Catal. 2017, 436, 128-137.

(S29) Gong, W.; Chen, C.; Zhang, H.; Wang, G.; Zhao, H. In Situ Synthesis of Highly Dispersed Cu-Co Bimetallic Nanoparticles for Tandem Hydrogenation/Rearrangement of Bioderived Furfural in Aqueous-Phase. ACS Sustain. Chem. Eng. 2018, 6, 14919-14925.

(S30) Jia, P.; Lan, X.; Li, X.; Wang, T. Highly Active and Selective NiFe/SiO 2 Bimetallic Catalyst with Optimized Solvent Effect for the Liquid-Phase Hydrogenation of Furfural to Furfuryl Alcohol. ACS Sustain. ACS Sustain. Chem. Eng. 2018, 6, 13287-13295.

(S31) Hu, F.; Wang, Y.; Xu, S.; Zhang, Z.; Chen, Y.; Fan, J.; Yuan, H.; Gao, L.; Xiao, G. Efficient and Selective $\mathrm{Ni} / \mathrm{Al}_{2} \mathrm{O}_{3}-\mathrm{C}$ Catalyst Derived from Metal-Organic Frameworks for the Hydrogenation of Furfural to Furfuryl Alcohol. Catal. Letters 2019, 149, 2158-2168.

(S32) Zhou, X.; Feng, Z.; Guo, W.; Liu, J.; Li, R.; Chen, R.; Huang, J. Hydrogenation and Hydrolysis of Furfural to Furfuryl Alcohol, Cyclopentanone, and Cyclopentanol with a Heterogeneous Copper Catalyst in Water. Ind. Eng. Chem. Res. 2019, 58, 3988-3993. 